\title{
On the Elimination of Non-Resonance Harmonics
}

\section{Eugene Wayne ${ }^{\dagger}$}

Department of Mathematics, The Pennsylvania State University, University Park, Pennsylvania 16802, USA

\begin{abstract}
Given a weakly coupled Hamiltonian system with short range, one dimensional interactions, and any initial conditions a canonical change of variables is constructed which yields a new Hamiltonian consisting of three parts - an integrable term, a resonant term whose effects are localized in those regions of the system which give small denominators in the KolmogorovArnol'd-Moser iteration scheme and a non-resonant interaction term which is very small. (In particular, much, much smaller than our original interactions.) The conditions which allow such a transformation to be constructed are independent of the number of degrees of freedom in the system, as are the estimates on the size of the various terms. Thus, if the resonances are "sparsely" distributed through the system most of the sites in the transformed Hamiltonian behave essentially like an integrable system, at least for as long a time as the trajectory of the system lies within the region where the canonical transformation is defined. In subsequent work it is shown that this time is long, and once again independent of the number of degrees of freedom in the system.
\end{abstract}

\section{Introduction}

In the present paper we continue the study of Hamiltonian systems with short range interactions begun in [6]. We prove a theorem which we call the elimination of nonresonance harmonics, because of its similarity to the lemma of the same name in [5]. Roughly speaking our result is as follows. Take a Hamiltonian in action-angle coordinates with short range interactions, e.g.

$$
H(I, \phi)=\frac{1}{2}\langle I, I\rangle+\varepsilon \sum_{i=1}^{N-1} \cos \left(\phi_{i+1}-\phi_{i}\right),
$$

and $N$ degrees of freedom. Given some initial condition $\left(I_{0}, \phi_{0}\right)$ define a set of "primary resonance vectors of order zero" to be $v \in \mathbb{Z}^{N}$ such that the denominators, in the expression for the generating function of the canonical change of variables that

† Supported in part by NSF Grant DMS-8403664 
solves the Hamilton-Jacobi equation, corresponding to these $v$ 's are "very small." Given the Hamiltonian (1.1), the solution of the Hamilton-Jacobi equation given by classical perturbation theory is the change of variables with generating function $\left\langle I^{\prime}, \phi\right\rangle+S\left(I^{\prime}, \phi\right)$, where

$$
S\left(I^{\prime}, \phi\right)=\frac{\varepsilon}{2} \sum_{v} \frac{e^{i v \cdot \phi}}{i\left\langle I^{\prime}, v\right\rangle},
$$

and the sum over $v$ runs over all vectors of the form $(0, \ldots, 0, \pm 1, \mp 1,0, \ldots, 0)$. We are interested in $I^{\prime}$, near $I_{0}$, so we define the resonant vectors, $R$, to be those $v$ in (1.2) such that $\left|\left\langle I_{0}, v\right\rangle\right|\left\langle c_{0}\right.$, where $c_{0}$ is a constant determined in the course of the proof. We then define $S\left(I^{\prime}, \phi\right)$ by (1.2) but we restrict the sum over $v$ to those $v \notin R$. If we then make the canonical change of variables defined by this generating function (it will be well defined provided $\varepsilon$ is sufficiently small) we find that the Hamiltonian (1.1) is transformed into a Hamiltonian $H^{1}\left(I^{\prime}, \phi^{\prime}\right)=h^{1}\left(I^{\prime}\right)+f^{1, \text { resonant }}\left(I^{\prime}, \phi^{\prime}\right)+$ $f^{1, \text { nonresonant }}\left(I^{\prime}, \phi^{\prime}\right)$. The resonant part of the interaction, $f^{1, \text { resonant }}$, has a Fourier series $\sum f_{v}^{1}\left(I^{\prime}\right) e^{\mathrm{i} v \cdot \phi}$, which has non-zero contributions only if $v \in R$, or for those $v^{\prime}$ with $\operatorname{supp} v^{\prime}$ "close" to supp $v$, for some $v \in R$. Since our change of variables was forced to ignore the contributions of the resonant vectors, we expect $f^{1, \text { resonant }}$ will still be $\mathcal{O}(\varepsilon)$. On the other hand, we have chosen $S\left(I^{\prime}, \phi\right)$ so that the change of variables will "kill" the $\mathcal{O}(\varepsilon)$ terms in the non-resonant interaction. Thus, we expect $f^{1 \text {,nonresonant }} \sim \mathcal{O}\left(\varepsilon^{2}\right)$. We now iterate this procedure (a finite number of times) and we find that there is a canonical change of variables $C$, such that

$$
\tilde{H}(I, \phi)=H \circ C(I, \phi)=\tilde{h}\left(I^{\prime}\right)+\widetilde{f}^{\text {resonant }}\left(I^{\prime}, \phi^{\prime}\right)+\widetilde{f}^{\text {nonresonant }}\left(I^{\prime}, \phi^{\prime}\right),
$$

where the Fourier series of $f^{\text {resonant }}$ contains the resonant harmonics we encountered at the various steps in the iterative procedure while $f^{\text {nonresonant }} \sim \mathcal{O}\left(e^{-1 / \varepsilon^{a}}\right)$ for some positive constant $a$. The conditions which allow this change of variables to be constructed (and also the constant $a$ ) are independent of the number of degrees of freedom in the system.

This would be of little interest if all vectors, $v$, were resonant. However, in [8] we demonstrate that for "typical" initial conditions, $\left(I_{0}, \phi_{0}\right)$, the resonant vectors are quite "sparse" and, hence, that the motion of the system is governed largely by $f^{\text {nonresonant }}$. We then show that this implies that for most sites $j$ in the system, the motion of $I_{j}(t)$ is indistinguishable from that of an integrable system for a long (but finite) period of time. Thus, while the system may well become ergodic as the number of degrees of freedom $N \rightarrow \infty$, the irregular motion tends to be localized in the vicinity of the resonances, (at least for $\varepsilon$ small), while large parts of the system undergo very regular motion, for a long time.

To state our results more precisely we introduce some notation. Let $V$ be a set in $\mathbb{R}^{N}$. A Hamiltonian in action angle form is a function $H^{0}(I, \phi): V \times \mathbb{R}^{N} \rightarrow \mathbb{R}$ that is periodic with period $2 \pi$ in each of the $\phi_{j}$ variables. Since $H^{0}$ is periodic as a function of $\phi$ we may also regard $H^{0}(I, \phi)$ as a function on $V \times T^{N}$, where $T^{N}$ is the $N$-torus. We will make no distinction between these two meanings for $H^{0}(I, \phi)$. Write the Hamiltonian as

$$
H^{0}(I, \phi)=h^{0}(I)+f^{0}(I, \phi)
$$


where $f^{0}(I, \phi)$ is assumed to be small, in a sense made precise below. Given an $N$ vector $\rho^{0}$, and a constant $\xi_{0}$ we define the domain $W\left(\rho^{0}, \xi_{0} ; V\right) \equiv \bigcup_{I \in V}$ $\left\{\left(I^{\prime}, \phi\right) \in \mathbb{C}^{2 N}|| \operatorname{Im} \phi_{i}\left|<\xi_{0},\right| I_{i}^{\prime}-I_{i} \mid<\rho_{i}^{0}\right.$, for $\left.i=1, \ldots, N\right\}$. We assume that there are constants $\rho_{0}$ and $\xi_{0}$ such that $H^{0}(I, \phi)$ is analytic on $W\left(\rho^{0}, \xi_{0} ; V\right)$, with $\rho^{0}=$ $\rho_{0}(1, \ldots, 1)$. Since we need lots of analyticity in the angular variables to prove our main theorem we assume $\xi^{0} \gg 1$. For later convenience define $\operatorname{pr}_{1} W\left(\rho^{0}, \xi_{0} ; V\right)$ to be $\left\{I \in \mathbb{R}^{N} \mid \exists \phi \in \mathbb{R}^{N}\right.$ such that $(I, \phi) \in W\left(\rho^{0}, \xi_{0} ; V\right)$. $\}$. Given initial conditions $\left(I_{0}, \phi_{0}\right)$ define the strength of the interaction, $\varepsilon_{0}$, by

$$
\sup \left\{\left|\frac{\partial f^{0}}{\partial I_{j}}\right|+\rho_{0}^{-1}\left|\frac{\partial f^{0}}{\partial \phi_{j}}\right|\right\} \leqq \varepsilon_{0},
$$

where the supremum runs over both $j=1, \ldots, N$ and $(I, \phi) \in W\left(\rho^{0}, \xi_{0} ;\left\{I_{0}\right\}\right)$. We note that it might seem more natural to choose $\varepsilon$ to measure the strength of the interaction in (1.1), but a little calculation shows that $\varepsilon$ and $\varepsilon_{0}$ are related by $\varepsilon \rho_{0}^{-1} e^{2 \xi_{0}} \leqq \varepsilon_{0}$, and (1.5) proves somewhat more useful in the general case.

The Hamiltonians we consider consist of almost independent almost identical subsystems, lying along a line, with interactions which decrease rapidly in strength as the distance between the points of interaction increases.

(a) nearly independent, nearly identical subsystems: Define $\omega^{0}(I)=\partial h / \partial I(I)$. We require

$$
\sup \left|\frac{\partial \omega_{i}^{0}}{\partial I_{j}}\right| \leqq e^{-m|i-j|} \quad \text { if } i \neq j,
$$

for some constant $m>0$, and

$$
\frac{\partial \omega_{i}^{0}}{\partial I_{i}}(I)=1+\chi^{0}(I ; i, i)
$$

with $\sup \left|\chi^{0}(I ; i, i)\right| \leqq c_{1}, c_{1}$ some universal constant, say $2^{-3}$. The suprema all run over $W\left(\rho^{0}, \xi^{0} ;\left\{I_{0}\right\}\right)$.

(b) short range interactions:

$$
\begin{aligned}
& \sup \left|\frac{\partial^{2} f^{0}}{\partial \phi_{i} \partial \phi_{j}}(I, \phi)\right| \leqq \rho_{0}^{2} e^{-m|--j|}, \\
& \sup \left|\frac{\partial^{2} f^{0}}{\partial \phi_{i} \partial I_{j}}(I, \phi)\right| \leqq \rho_{0} e^{-m|i-j|}, \\
& \sup \left|\frac{\partial^{2} f^{0}}{\partial I_{i} \partial I_{j}}(I, \phi)\right| \leqq e^{-m|i-j|} .
\end{aligned}
$$

All suprema are taken over $W\left(\rho^{0}, \xi_{0} ;\left\{I_{0}\right\}\right)$. This definition of short range interactions differs slightly from that of [6]. Except for the factors of $\rho_{0}$, however, which merely serve to keep the dimensions of the two sides the same, (and the corresponding factors of $\varepsilon_{0} \rho_{0}, \varepsilon_{0}$, and $\varepsilon_{0} \rho_{0}^{-1}$ in [6]) the previous definition follows from (1.8) by applying Cauchy's theorem and setting $m=\left|\ln \left(\varepsilon_{0} \rho_{0}^{-1}\right)\right|$. Note also that (1.1) obeys (1.8) provided we choose $\varepsilon$ such that $\varepsilon e^{2 \xi_{0}}=\rho_{0}^{2} e^{-m}$. 
We note that the conditions above correspond to considering this system as lying on a one dimensional lattice. We expect our results to extend to higher dimensional lattices, and to systems with periodic boundary conditions, but the technical difficulties encountered are greater there.

Theorem 1.1. (The elimination of non-resonance harmonics) There exist universal constants $0<c \ll 1,0<\sigma$, and $K \gg 1$, and a constant $k_{0}$ defined below such that if

$$
\varepsilon_{0}<c \rho_{0}\left(k_{0}\right)^{-\sigma} \text { and } m>K,
$$

we can construct a set, $R$, of vectors $v \in \mathbb{Z}^{N}$, an $N$ vector $\tilde{\rho}$ and a change of variables $C:\left(I^{\prime}, \phi^{\prime}\right) \rightarrow(I, \phi)$, analytic and invertible on $W(\tilde{\rho}, 1 ;\{\widetilde{I}\})$, where $\tilde{I}$ will be defined in the course of the proof. Furthermore, if $\Gamma=p r_{1} W(\tilde{\rho}, 1,\{\widetilde{I}\}), C$ is canonical on $\Gamma \times T^{N}$. Defining $\tilde{H}\left(I^{\prime}, \phi^{\prime}\right)=H^{0} \circ C\left(I^{\prime}, \phi^{\prime}\right)$, we have

$$
\tilde{H}\left(I^{\prime}, \phi^{\prime}\right)=\tilde{h}\left(I^{\prime}\right)+\widetilde{f}\left(I^{\prime}, \phi^{\prime}\right)=\tilde{h}\left(I^{\prime}\right)+\widetilde{f}^{\text {resonant }}\left(I^{\prime}, \phi^{\prime}\right)+\tilde{f}^{\text {nonresonant }}\left(I^{\prime}, \phi^{\prime}\right),
$$

with $f^{\text {resonant }}\left(I^{\prime}, \phi^{\prime}\right)=\sum_{v \in R} \widetilde{f}_{v}\left(I^{\prime}\right) e^{i v \cdot \phi}$. The interactions of $H\left(I^{\prime}, \phi^{\prime}\right)$ obey the bounds

$$
\begin{aligned}
& \sup \left|\frac{\partial^{2} \tilde{f}}{\partial \phi_{i}^{\prime} \partial \phi_{j}^{\prime}}\left(I^{\prime}, \phi^{\prime}\right)\right| \leqq \rho_{0}^{2} e^{-(3 m / 4)|\imath-j|}, \\
& \sup \left|\frac{\partial^{2} \widetilde{f}}{\partial \phi_{i}^{\prime} \partial I_{j}^{\prime}}\left(I^{\prime}, \phi^{\prime}\right)\right| \leqq \rho_{0} e^{-(3 m / 4)|i-j|} \\
& \sup \left|\frac{\partial^{2} f}{\partial I_{i}^{\prime} \partial I_{j}^{\prime}}\left(I^{\prime}, \phi^{\prime}\right)\right| \leqq e^{-(3 m / 4)|\imath-j|}
\end{aligned}
$$

and

$$
\begin{aligned}
& \sup \left\{\left|\frac{\partial \tilde{f}_{\text {nonresonant }}}{\partial I_{j}^{\prime}}\left(I^{\prime}, \phi^{\prime}\right)\right|+\rho_{0}^{-1}\left|\frac{\partial \tilde{f}^{\text {nonresonant }}}{\partial \phi_{j}^{\prime}}\left(I^{\prime}, \phi^{\prime}\right)\right|\right\} \\
& \quad \leqq \rho_{0}\left(\varepsilon_{0} \rho_{0}^{-1}\right)^{(3 / 2)^{k_{0}}},
\end{aligned}
$$

for all $i$ and $j$, with the suprema running over $W(\tilde{\rho}, 1 ;\{\widetilde{I}\})$. The constant $k_{0}$ in (1.12) is given by $k_{0} \leqq \min \left(\left[\left(\xi_{0}-1\right) / K_{1}\right],\left[m / K_{1}\right]\right)$, for $K_{1}$ some universal constant which could be calculated from the proof of the theorem, and $[x]=$ integer part of $x$. (We remark that one could make the interactions in (1.11) decay as $e^{-(1-\delta) m|i-j|}$, for any $0<\delta<1$, by slight modifications in the proof, and by changing the constants in (1.9).) We note that this theorem can be trivially proved by picking $R$ to be all of $\mathbb{Z}^{N}$, in which case, $f^{\text {nonresonant }}=0$, and we have gained nothing. The point is, that the procedure used to define $R$ yields, for most initial conditions, a "small" set of vectors $R$, so that the effects $\tilde{f}^{\text {resonant }}$ are localized, and the motion of the system is largely determined by $\tilde{h}+\tilde{f}^{\text {nonresonant }}$. This, as we demonstrate in [8], allows one to give strong bounds on the trajectories of the system.

The proof of this theorem can be used to give bounds on the amount by which the canonical transformation $C$ differs from the identity. Such estimates are implicit in Sects. 3 and 4.

We now discuss the application of this theorem to the Hamiltonian (1.1). Since 
the Hamiltonian is an entire function we can take the size of the analyticity domain as large as we like. Choose $\xi_{0}=\frac{1}{4}|\ln \varepsilon|$ and $\rho_{0}=1$. Then condition (1.5) implies $\varepsilon e^{2 \xi_{0}}=$ $\varepsilon^{1 / 2} \leqq \varepsilon_{0}$. Set $\varepsilon_{0}=\varepsilon^{1 / 2}$. Then the left hand side of (1.8) is bounded by $\varepsilon e^{2 \xi_{0}}=\varepsilon^{1 / 2} \leqq$ $e^{-m}$. If we set $m=\frac{1}{2}|\ln \varepsilon|=\left|\ln \varepsilon_{0}\right|$, then (1.8) is satisfied and by making $\varepsilon$ small we can make $m$ as large and $\varepsilon_{0}$ as small as needed. Furthermore, the constant $k_{0}$ in Theorem 1.1 can be chosen to be $k_{0}=\left[\left(1 / 5 K_{1}\right)|\ln \varepsilon|\right]$. Finally note that $(1.6)$ and (1.7) are trivially satisfied for (1.1). Thus we obtain as a corollary to Theorem 1.1:

Corollary 1.2. Given the Hamiltonian (1.1) there is some constant $0<c \ll 1$ (independent of $N$ ) such that if $0 \leqq \varepsilon<c$ we may construct a canonical transformation $C, b y$ Theorem 1.1 such that

$$
H \circ C\left(I^{\prime}, \phi^{\prime}\right)=\tilde{h}\left(I^{\prime}\right)+\tilde{f}^{\text {resonant }}\left(I^{\prime}, \phi^{\prime}\right)+\widetilde{f}^{\text {nonresonant }}\left(I^{\prime}, \phi^{\prime}\right)
$$

with

$$
\sup \left\{\left|\frac{\partial \tilde{f}^{\text {nonresonant }}}{\partial I_{j}^{\prime}}\left(I^{\prime}, \phi^{\prime}\right)\right|+\left|\frac{\partial \widetilde{f}^{\text {nonresonant }}}{\partial \phi_{j}^{\prime}}\left(I^{\prime}, \phi^{\prime}\right)\right|\right\} \leqq \varepsilon^{(1 / 2)(3 / 2)^{k_{0}}} \leqq e^{-1 / \varepsilon^{a}}
$$

for some positive constant a, (independent of the number of degrees of freedom of the system.) The supremum runs over $W\left(\tilde{\rho}, 1 ;\left\{\tilde{I}_{0}\right\}\right)$ (with $\tilde{\rho}$ and $\tilde{I}$ defined in the course of the proof.) Also, the interactions $7^{\text {resonant }}+f^{\text {nonresonant, }}$ satisfy (1.11) with $m=\frac{1}{2}|\ln \varepsilon|$.

We close this section with some remarks about notation. Throughout the paper $B, B^{\prime}, B_{1}, B_{2}, \ldots$ will denote universal constants of magnitude greater than one, while $c, c^{\prime}, c_{1}, c_{2}, \ldots$ will denote constants of magnitude less than one. They may represent different constants in different contexts.

The second note concerns the factors of $\rho_{0}$ which occur, for instance, in (1.8) and (1.11). These are included to keep the dimensions of the two sides of the inequality the same, since as was pointed out in [4], this often provides a check on one's calculations at intermediate stages of the proof. In the final analysis, however, they are less important and we will often, to save space, write inequalities like (1.8) as follows: Let $x_{i}$ equal either $I_{i}$ or $\phi_{i}$ depending on the context. Then $f^{0}(I, \phi)=f^{0}(x)$ and we write (1.8) as

$$
\sup \left|\frac{\partial^{2} f^{0}}{\partial x_{i} \partial x_{j}}(x)\right| \leqq \rho_{0}^{n} e^{-m|i-j|},
$$

where $n$ is chosen to keep the dimensions of the two sides of the inequality the same. If the reader finds this notation ambiguous just set $\rho_{0}^{n}=K$ wherever it appears and remember that $K$ is a constant depending on the size of the analyticity domain of the initial Hamiltonian, but independent of the number of degrees of freedom in the system.

We note that Theorem 1.1 is related to Theorem 1.1 of [6]. There are two principal differences in the results. First of all, by more careful estimates it has proved possible to eliminate all dependence on the number of degrees of freedom of the system. This is largely a technical improvement. Of more fundamental importance is the fact that the present work allows resonant regions to exist in the system, but provides a method of isolating (at least for a finite time) their effects. The work of Nekhoroshev [5] provided the motivation for this improvement in the theory. 
A final note concerning terminology - when we speak of "dimensional estimates" we mean the standard estimates on derivatives of analytic functions that Cauchy's theorem provides.

Recently, Benettin et al. [1] have studied the model (1.1) by means of classical perturbation theory. They have shown that one can construct a canonical transformation which transforms (1.1) into an integrable system up to errors of arbitrarily high order in $\varepsilon$ by this means. Their method (like that of the present work) is restricted to small $\varepsilon$, but thus far the dependence of the size of the allowed perturbation on the parameters of the system, such as the number of degrees of freedom, has not been determined. If these estimates are performed their method may yield an alternate proof of Corollary 1.2.

\section{The Induction Procedure}

Consider the initial Hamiltonian $H^{0}(I, \phi)$ of Sect. 1. We first locate the primary resonances of order zero, $R_{p}^{0}$. Let $\mathbb{X}_{0}=\left\{v \in \mathbb{Z}^{N}\left|d(\operatorname{supp} v) \leqq L_{0}, 0<\right| v \mid \leqq M_{0}\right\}$, where $d(\operatorname{supp} v)$ is the diameter of $\operatorname{supp} v$, if $v$ is considered as an integer valued function on the lattice $\{1, \ldots, N\},|v|=\sum_{j=1}^{N}\left|v_{j}\right|$, and $L_{0}$ and $M_{0}$ are constants defined below. Given initial conditions $\left(I_{0}, \phi_{0}\right)$, set $\omega^{0}(I)=\partial h^{0} / \partial I(I)$ and define

$$
R_{p}^{0}=\left\{v \in \mathbb{X}_{0}||\left\langle\omega^{0}\left(I_{0}\right), v\right\rangle \mid<\rho_{0} \lambda\left(\varepsilon_{0}\right)\left\{B_{1} e^{(3 / 2)|v|+L_{0}}\right\}^{-1}\right\},
$$

where $\lambda\left(\varepsilon_{0}\right)=\left(\varepsilon_{0} \rho_{0}^{-1}\right)^{\alpha}$ for $\alpha$ some small positive constant that will be implicitly defined in the course of the proof and $B_{1}$ some large constant. In the KAM theory one attempts to construct a canonical change of coordinates which "kills" the nonintegrable part of the Hamiltonian to order $\varepsilon_{0}^{2}$. In the present case we must be content to "kill" only those harmonics, (Fourier coefficients), $f_{v}^{0}(I)$, with $v \notin R_{p}^{0}$. Constructing a canonical transformation $C^{0}$, via the classical perturbation theory, we obtain a new Hamiltonian

$$
H^{1}\left(I^{\prime}, \phi^{\prime}\right)=H^{0} \circ C^{0}\left(I^{\prime}, \phi^{\prime}\right)=h^{1}\left(I^{\prime}\right)+f^{1, \mathrm{r}}\left(I^{\prime}, \phi^{\prime}\right)+f^{1, \mathrm{nr}}\left(I^{\prime}, \phi^{\prime}\right) .
$$

As expected, the size of $f^{1, n r}\left(I^{\prime}, \phi^{\prime}\right) \ll \varepsilon_{0}$. What is perhaps slightly surprising is that one must include in the "resonant" part of the interaction, $f^{1, r}$, not only harmonics $f_{v}^{1}(I)$ with $v \in R_{p}^{0}$, but also those harmonics which are "close" to primary resonances, in a sense made precise below. We call these harmonics the secondary resonances of zeroth order. One then iterates this procedure locating at each stage the primary resonances, and choosing the canonical transformation to kill the nonresonant harmonics. A finite number of such iterations suffices to prove Theorem 1.1.

Suppose we have constructed $H^{k}(I, \phi)=h^{k}(I)+f^{k, \mathrm{r}}(I, \phi)+f^{k, \mathrm{nr}}(I, \phi)$, with $f^{k, \mathrm{r}}(I, \phi)=\sum_{v \in \tilde{R}^{k}} f_{v}^{k}(I) e^{i v \cdot \phi}$, for some set of harmonics $\tilde{R}^{k}$, specified below. Define $L_{k}=2(3 / 2)^{k}\left|\ln \left(\varepsilon_{0} \rho_{0}^{-1}\right)\right| / m, M_{k}=(3 / 2)^{k}\left|\ln \left(\varepsilon_{0} \rho_{0}^{-1}\right)\right| / \delta$, for $\delta$ a universal constant to be specified in the course of the proof. Define $\rho_{k+1}=\rho_{k} \lambda\left(\varepsilon_{0}\right)\left[B_{2} e^{2 M_{k}+2 L_{k}}\right]^{-1}$, for $k=0, \ldots$, and set $\mathbb{X}_{k}=\left\{v \in \mathbb{Z}^{N}\left|d(\operatorname{supp} v) \leqq L_{k}, 0<\right| v \mid \leqq M_{k}\right\}$. Let $\omega^{k}(I)=$ $\left(\partial h^{k} / \partial I\right)(I)$. In the course of the proof we will define a sequence of vectors $I_{0}$, $I_{1}, \ldots, I_{k_{0}}$, whose first element is the initial value of the action variables, $I$. The 
primary resonances of order $k$ are

$$
R_{p}^{k}=\left\{v \in \mathbb{X}_{k} \backslash \tilde{R}^{k}||\left\langle\omega^{k}\left(I_{k}\right), v\right\rangle \mid<\rho_{0} \lambda\left(\varepsilon_{0}\right)\left[B_{1} e^{(3 / 2)|v|+L_{k}}\right]^{-1}\right\} .
$$

The secondary resonances of order $k$ are

$$
R_{s}^{k}=\left\{v \in \mathbb{X}_{k+1} \backslash\left(\tilde{R}^{k} \cup R_{p}^{k}\right) \mid \overline{\operatorname{supp} v} \cap \overline{\operatorname{supp} v^{\prime}} \neq \varnothing \text { for some } v^{\prime} \in \widetilde{R}^{k} \cup R_{p}^{k}\right\} .
$$

Here $\overline{\operatorname{supp} v}=\{i, i+1, \ldots, j-1, j\}$, for $i$ and $j$ respectively the leftmost and rightmost sites in $\operatorname{supp}_{k-1} v$. We assume that the set of vectors $\widetilde{R}^{k}$ which defines $f^{k, r}$ above is given by $\tilde{R}^{k}=\bigcup_{m=0}^{k-1}\left[R_{p}^{m} \cup R_{s}^{m}\right]$ if $k>0$, and $\tilde{R}^{0}=\varnothing$.

The $k^{\text {th }}$ order sites, $S^{k}$, are all sites $j$ such that:

(i) $j \in \overline{\operatorname{supp} v}$ for some $v \in R_{p}^{k} \cup R_{s}^{k-1}$ (for $k=0$ take $R_{s}^{-1} \equiv \varnothing$,)

(ii) $j \notin S^{m}, m=0, \ldots, k-1$.

Roughly speaking, the motion of $\left(I_{j}(t), \phi_{j}(t)\right)$ for $j \in S^{m}$, is controlled by the $m^{\text {th }}$ order resonances.

One interesting technical difference between the present work and previous work on the KAM theory is that we must allow the size of the neighborhood on which we define our change of variables to vary as we move about through the system. Given a domain $W(\rho, \xi ;\{I\}),(\rho)_{j}$ determines the size of the complex neighborhood about $I_{j}$, and we must choose that size to be much larger when $j \in S^{m}$, for $m$ small, than when $j$ lies in the non-resonant regions (i.e. $j \notin S^{m}, m=0, \ldots, k$ ). Define

$$
\left(b^{m}\right)_{i}=\left\{\begin{array}{l}
c_{1} \rho_{m+1} / k_{0} \quad \text { if } i \in S^{m}, m=0, \ldots, k \\
0 \text { otherwise }
\end{array}\right.
$$

Then for $k \geqq 1$, define

$$
\left(\rho^{k}\right)_{i}= \begin{cases}\rho_{k} \text { if } i \notin \bigcup_{m=0}^{k-2} S^{m} & \text { (or for all } i \text { if } k=1) \\ \left(\rho^{k-1}\right)_{i}-8\left(b^{m}\right)_{i} & \text { if } \quad i \in S^{m}, m=0, \ldots, k-2 .\end{cases}
$$

In the angular variables $\phi$ we give up a fixed amount of analyticity with each iteration and set $\xi^{k+1}=\xi^{k}-3(\delta+2)$.

We assume that $H^{k}(I, \phi)$ obeys the following estimates, on $W\left(\rho^{k}, \xi_{k} ;\left\{I_{k}\right\}\right)$.

$$
\sup \left\{\left|\frac{\partial f^{k, \mathrm{nr}}}{\partial I_{j}}\right|+\rho_{0}^{-1}\left|\frac{\partial f^{k, \mathrm{nr}}}{\partial \phi_{j}}\right|\right\} \leqq \varepsilon_{k},
$$

with $\varepsilon_{k}=\rho_{0}\left(\varepsilon_{0} \rho_{0}^{-1}\right)^{(3 / 2)^{k}}$, and

where

$$
\sup \left\{\left|\frac{\partial f^{k, \mathrm{r}}}{\partial I_{j}}\right|+\rho_{0}^{-1}\left|\frac{\partial f^{k, \mathrm{r}}}{\partial \phi_{j}}\right|\right\} \leqq A(k, j),
$$

$$
A(k, j)= \begin{cases}\rho_{0}\left(\varepsilon_{k} \rho_{k}^{-1}\right)^{\left(1-\eta_{k}\right)} & \text { if } j \notin \bigcup_{n=0}^{k-1} S^{n} \\ \rho_{0}\left(\varepsilon_{n} \rho_{n}^{-1}\right)^{\left(1-\eta_{k}\right)} & \text { if } j \in S^{n}, n=0, \ldots, k-1,\end{cases}
$$


and $\eta_{k}=8 \sum_{j=0}^{k-1} \beta_{j},\left(\eta_{0}=0\right)$ and $\beta_{j}=c_{1}(3 / 2)^{-j}+c_{2} / k_{0}$, with $c_{1}$ and $c_{2}$ chosen so that $\eta_{k}<1 / 8$, for $k=0, \ldots, k_{0}$. We note that $\varepsilon_{k} \leqq A(k, j)$, so the non-resonant part of the interaction is smaller than the resonant part. We also assume that our Hamiltonian retains its short range character, so that

$$
\frac{\partial^{2} h^{k}}{\partial I_{I} \partial I_{j}}(I)=\delta_{i j}+\chi^{k}(I ; i, j),
$$

where

$$
\sup \left|\chi^{k}(I ; i, j)\right| \leqq \theta(k ; i, j)= \begin{cases}e^{-\left(1-\eta_{k}\right) m|i-j|} & \text { if } i \neq j \\ c_{1}+B k_{0} \sum_{j=0}^{k-1} \varepsilon_{j} \rho_{j+1}^{-1}, & \text { if } i=j\end{cases}
$$

and the constant $c_{1}$ that appears on the right-hand side of (2.9) is the same as that appearing in the bound on $\chi^{0}(I ; i, i)$ in Sect. 1 . We also need

$$
\sup \left|\frac{\partial^{2} f^{k}}{\partial x_{i} \partial x_{j}}(x)\right| \leqq \rho_{0}^{n} e^{-\left(1-\eta_{k}\right) m|\imath-\jmath|},
$$

with $x$ defined as in (1.14) and $\rho_{0}^{n}$ chosen to insure that the dimensions of the two sides of (2.10) are the same. Also, in both (2.9) and (2.10) the suprema run over $W\left(\rho^{k}, \xi_{k} ;\left\{I_{k}\right\}\right)$. Given these assumptions we have

Proposition 2.1. Let $H^{k}(I, \phi)$ be as above (with $\left.k=0,1, \ldots, k_{0}-1\right)$. Then if

$$
\varepsilon_{0}<c \rho_{0}\left(k_{0}\right)^{-\sigma} \text { and } m>K \text {, }
$$

with $c, \sigma, K$ the same constants as in Theorem 1.1, there exists a change of variables $C^{k}:\left(I^{\prime}, \phi^{\prime}\right) \rightarrow(I, \phi)$, analytic and invertible on $W\left(\rho^{k+1}, \xi_{k+1} ;\left\{I_{k+1}\right\}\right)$. Furthermore $C^{k}: W\left(\rho^{k+1}, \xi_{k+1} ;\left\{I_{k+1}\right\}\right) \rightarrow W\left(\rho^{k}, \xi_{k} ;\left\{I_{k}\right\}\right)$. If $\Gamma^{k+1}=\operatorname{pr}_{1} W\left(\rho^{k+1}, \xi_{k+1} ;\left\{I_{k+1}\right\}\right), C^{k}$ is canonical on $\Gamma^{k+1} \times T^{N}$. Define

$$
\begin{aligned}
H^{k+1}\left(I^{\prime}, \phi^{\prime}\right) & =H^{k} \circ C^{k}\left(I^{\prime}, \phi^{\prime}\right)=h^{k+1}\left(I^{\prime}\right)+f^{k+1}\left(I^{\prime}, \phi^{\prime}\right) \\
& =h^{k+1}\left(I^{\prime}\right)+f^{k+1, r}\left(I^{\prime}, \phi^{\prime}\right)+f^{k+1, n r}\left(I^{\prime}, \phi^{\prime}\right) .
\end{aligned}
$$

where $f^{k+1, r}\left(I^{\prime}, \phi^{\prime}\right)=\sum_{v \in R^{k} \cup R_{p}^{k} \cup R_{s}^{k}} f_{v}^{k+1}\left(I^{\prime}\right) e^{i v \cdot \phi^{\prime}}$. (The procedure for splitting $H^{k+1}\left(I^{\prime}, \phi^{\prime}\right)$ into its integrable $\left(h^{k+1}\right)$ and nonintegrable parts is given in Sect. 3.) $H^{k+1}\left(I^{\prime}, \phi^{\prime}\right)$ obeys the bounds (2.5)-(2.10), with $k$ replaced by $k+1$, and the suprema are now taken over $W\left(\rho^{k+1}, \xi_{k+1} ;\left\{I_{k+1}\right\}\right)$.

We note that $I_{k}$ is defined inductively by $\left(I_{k+1}, \phi_{k+1}\right)=\left(C^{k}\right)^{-1}\left(I_{k}, \phi_{k}\right)$. (Of course we must check in the course of the proof that $\left(1_{k}, \phi_{k}\right)$ lies in the domain of $\left(C^{k}\right)^{-1}$.)

Given Proposition 2.1 we immediately obtain Theorem 1.1. Note that our original Hamiltonian $H^{0}(I, \phi)$ satisfies $(2.5)-(2.10)$ if we take $\widetilde{R}^{0}=\varnothing$, and hence $f^{0, \mathrm{r}}(I, \phi)=0$. Now apply Proposition 2.1, until $k=k_{0}-1$. Note that if the constant $K_{1}$ in Theorem 1.1 is large enough (in particular $\left.K_{1}>3(\delta+2)\right) \xi_{k_{0}} \geqq 1$. Define $C=C^{0} \circ C^{1} \circ \ldots \circ C^{k_{0}-1}, \tilde{I}=I_{k_{0}}$, and $\tilde{\rho}=\rho_{k_{0}}$. Then $C$ is defined on $W(\tilde{\rho}, 1 ;\{\widetilde{I}\})$ and 
maps this set into $W\left(\rho, \xi_{0} ;\left\{I_{0}\right\}\right)$ so

$$
\begin{aligned}
\tilde{H}\left(I^{\prime}, \phi^{\prime}\right) & =H^{0} \circ C\left(I^{\prime}, \phi^{\prime}\right)=H^{k_{0}}\left(I^{\prime}, \phi^{\prime}\right) \\
& =\tilde{h}\left(I^{\prime}\right)+\widetilde{f}^{\text {resonant }}\left(I^{\prime}, \phi^{\prime}\right)+\tilde{f}^{\text {nonresonant }}\left(I^{\prime}, \phi^{\prime}\right),
\end{aligned}
$$

with $f^{\text {resonant }}\left(I^{\prime}, \phi^{\prime}\right)=f^{k_{0}, \mathrm{r}}\left(I^{\prime}, \phi^{\prime}\right)$ and $f^{\text {nonresonant }}\left(I^{\prime}, \phi^{\prime}\right)=f^{k_{0}, \mathrm{nr}}\left(I^{\prime}, \phi^{\prime}\right)$. The stated bounds on $\tilde{H}\left(I^{\prime}, \phi^{\prime}\right)$ then follow from (2.5)-(2.10).

Note that we obtain somewhat more information from Proposition 2.1 than was stated in Theorem 1.1 For instance from (2.6) and (2.7) we see that if $\delta$ and $m$ are large enough, $\varepsilon_{n} \rho_{n}^{-1} \leqq\left(\varepsilon_{0} \rho_{0}^{-1}\right)^{(3 / 4)(3 / 2)^{n}}(\delta$ and $m$ do not need to depend on $n$ for this to be the case,) and we have

Corollary 2.2. If $\tilde{H}(I, \phi)$ is the Hamiltonian constructed in Theorem 1.1 , then

$$
\begin{aligned}
& \sup \left\{\left|\frac{\partial f^{\text {resonant }}}{\partial I_{j}}\right|+\rho_{0}^{-1}\left|\frac{\partial \tilde{f}^{\text {resonant }}}{\partial \phi_{j}}\right|\right\} \\
& \leqq \begin{cases}\rho_{0}\left(\varepsilon_{0} \rho_{0}^{-1}\right)^{(1 / 2)(3 / 2)^{k_{0}}} & \text { if } j \notin \bigcup_{n=0}^{k_{0}-1} S^{n} \\
\rho_{0}\left(\varepsilon_{0} \rho_{0}^{-1}\right)^{(1 / 2)(3 / 2)^{n}} & \text { if } j \in S^{n}, n=0, \ldots, k_{0}-1,\end{cases}
\end{aligned}
$$

and the supremum runs over $W(\tilde{\rho}, 1 ;\{\widetilde{I}\})$.

We remark that at many places in the proof we will state inequalities which are true, provided $m$ and $\delta$ are sufficiently large (where "sufficiently large" does not depend on the number of degrees of freedom of the system) without explicitly stating this assumption. Note also, that because of the definition of $m, k_{0}$, and $\beta_{k}$, $m \cdot \beta_{k} \geqq c_{2} K_{1}$, for all $k=0, \ldots, k_{0}$, where $K_{1}$ is some universal constant which we may choose as large as we need, which means that $m \cdot \beta_{k}$ can be chosen to be some large constant.

We note that one could replace the factors of $(1 / 2)$ in the exponents in (2.14) with any number less than one, by making only minor changes in the proof.

\section{The Canonical Transformation}

In this section we construct the canonical transformation, $C^{k}$, whose existence is asserted in Proposition 2.1. Let $H^{k}(I, \phi)=h^{k}(I)+f^{k}(I, \phi)$ be a Hamiltonian satisfying the inductive hypotheses of the previous section. Define the generating function for the desired change of variables by

$$
S\left(I^{\prime}, \phi\right)=\sum_{\substack{v \in \mathbb{X}_{k} \\ v \notin \tilde{R}^{k} \cup R_{p}^{k}}} \frac{f_{v}^{k}\left(I^{\prime}\right) e^{i v \cdot \phi}}{i\left\langle\omega^{k}\left(I^{\prime}\right), v\right\rangle},
$$

with $f_{v}^{k}\left(I^{\prime}\right)$ the Fourier coefficients of $f^{k}\left(I^{\prime}, \phi^{\prime}\right)$.

This is the generating function that one is led to by classical perturbation theory if one only attempts to "kill" the non-resonant harmonics in the interactions. Since there are only a finite number of terms in the definition of $S$, it can fail to be well defined only if the denominator of one of the terms vanishes. That this does not occur is guaranteed by 
Lemma 3.1. Define the N-vector $r_{k}^{n}$ by $\left(r_{k}^{n}\right)_{i}=2^{8-n} \rho_{k+1}$ if $i \notin \bigcup_{m=0}^{k-2} S^{m},\left(r_{k}^{n}\right)_{i}=\left(\rho^{k}\right)_{i}-$ $n\left(b^{m}\right)_{i}$ if $i \in S^{m}, m=0, \ldots, k-2$. Then on $W\left(r_{k}^{1}, \xi_{k} ;\left\{I_{k}\right\}\right)$,

$$
\sup \left[\left|\left\langle\omega^{k}\left(I^{\prime}\right), v\right\rangle\right|^{-1}\right] \leqq 2 B_{1}\left(\rho_{0} \lambda\left(\varepsilon_{0}\right)\right)^{-1} \exp \left[(3 / 2)|v|+L_{k}\right],
$$

for all $v \in \mathbb{X}_{k}$, but $v \notin \widetilde{R}^{k} \cup R_{p}^{k}$. The constant $B_{1}$ in this inequality is the same as that in (2.1).

Remark. We could actually define the generating function $S$ on a larger domain than $W\left(r_{k}^{1}, \xi_{k} ;\left\{I_{k}\right\}\right)$. If $R_{p}^{k}$ is defined by $(2.1)$, let $V_{k}$ be the largest connected set in $\mathbb{R}^{N}$ containing $I_{k}$, such that if $v \in \mathbb{X}_{k} \backslash\left(\widetilde{R}^{k} \cup R_{p}^{k}\right)$, and $I \in V_{k}, \quad\left|\left\langle\omega^{k}(I), v\right\rangle\right|^{-1} \leqq$ $B_{1}\left(\rho_{0} \lambda\left(\varepsilon_{0}\right)\right) \exp \left[(3 / 2)|v|+L_{k}\right]$. (Roughly speaking $V_{k}$ is the set of $I$ 's with the same resonant vectors as $\left.I_{k}\right)$. Then (3.2) holds on the larger domain $W\left(r_{k}^{1}, \xi_{k} ; V_{k}\right)$, with an attendant increase in the size of the domains on which the canonical transformations in Proposition 2.1 and Theorem 1.1 are defined. At present, however, I have found no use for this larger domain.

Proof. Since $v \in \mathbb{X}_{k}, v \notin \widetilde{R}^{k} \cup R_{p}^{k}$,

$$
\left|\left\langle\omega^{k}\left(I_{k}\right), v\right\rangle^{-1}\right| \leqq B_{1}\left(\rho_{0} \lambda\left(\varepsilon_{0}\right)\right)^{-1} \exp \left[(3 / 2)|v|+L_{k}\right] .
$$

Furthermore there is a path $\gamma$, consisting of $N$ components, $\gamma_{j}$, along which only one coordinate of $I$ varies, joining $I_{k}$ to $I^{\prime}$ for every $I^{\prime}$ such that $\left(I^{\prime}, \phi\right)$ is in $W\left(r_{k}^{1}, \xi_{k} ;\left\{I_{k}\right\}\right)$ for some $\phi$. Also, the length of $\gamma_{j}$ is bounded by $\left(r_{k}^{1}\right)_{j}$. By the fundamental theorem of calculus,

$$
\left\langle\omega^{k}\left(I^{\prime}\right), v\right\rangle^{-1}=\left\langle\omega^{k}\left(I_{k}\right), v\right\rangle^{-1}\left\{1+\left\langle\omega^{k}\left(I_{k}\right), v\right\rangle^{-1} \times \int_{\gamma} d I^{\prime \prime}\left\langle\frac{\partial \omega^{k}}{\partial I}\left(I^{\prime \prime}\right), v\right\rangle\right\}^{-1} .
$$

Since $v \notin \widetilde{R}^{k} \cup R_{p}^{k},\left(r_{k}^{1}\right)_{j}=2^{7} \rho_{k+1}$ for all $j$ such that $\operatorname{dist}(j, \operatorname{supp} v) \leqq L_{k-1}$. (If $k=0$ or 1 , $\left(r_{k}^{1}\right)_{j}=2^{7} \rho_{k+1}$ for all sites $j$.) This follows since if $j \in \bigcup_{m=0}^{k-2} S^{m}$, the definition of the secondary resonances would force $v$ to be an element of $R_{s}^{k-1}$ if $\operatorname{dist}(j, \operatorname{supp} v) \leqq$ $L_{k-1}$. Write

$$
\int_{\gamma} d I^{\prime \prime}\left\langle\frac{\partial \omega^{k}}{\partial I}\left(I^{\prime \prime}\right), v\right\rangle=\sum_{i, j} \int_{\gamma_{j}} \frac{\partial^{2} h^{k}}{\partial I_{i} \partial I_{j}}\left(I^{\prime \prime}\right) v_{i} d I_{j}^{\prime \prime}
$$

For each $i \in \operatorname{supp} v$, bound the sum over $j$ by breaking it into two parts. For those $j$ 's with $|j-i| \leqq L_{k-1}$, bound the length of $\gamma_{j}$ by $2^{7} \rho_{k+1}$, while for $j$ 's with $|j-i|>L_{k-1}$ we bound the length of $\gamma_{j}$ by $\rho_{0}$. In all cases, the integrand is bounded by (2.8) and (2.9). Summing the resulting geometric series in $j$ we find (3.5) is bounded in magnitude by

$$
2^{8}|v| \rho_{k+1}+2^{2}|v| \rho_{0} e^{-\left(1-\eta_{k}\right) m L_{k-1}},
$$

(provided $m$ is sufficiently large). Bounding the factor of $\left\langle\omega^{k}\left(I_{k}\right), v\right\rangle^{-1}$ by (3.3), and using the fact that $|v| \leqq M_{k}$ since $v \in \mathbb{X}_{k}$, we see that the quantity in $\{\cdots\}$ in (3.4) can be bounded below by (1/2). Then Lemma 3.1 follows. (If $k=0$ or 1 , the second term in (3.6) may be omitted and Lemma 3.1 still follows.) 
Lemma 3.1 allows us to bound the derivatives of $S\left(I^{\prime}, \phi\right)$. We obtain the following results which we prove in Sect. 7. To simplify our notation let $y_{i}$ be either $I_{i}^{\prime}$ or $\phi_{i}$ depending on the context. Then regard $S\left(I^{\prime}, \phi\right)=S(y)$.

Proposition 3.2. On $W\left(r_{k}^{1}, \xi^{k}-\delta ;\left\{I_{k}\right\}\right)$,

$$
\sup \left|\frac{\partial S}{\partial y_{i}}(y)\right| \leqq \rho_{0}^{n}\left(\lambda\left(\varepsilon_{0}\right)\right)^{-2} \varepsilon_{k} B^{L_{k}}
$$

and on $W\left(r_{k}^{2}, \xi^{k}-\delta-1 ;\left\{I_{k}\right\}\right)$,

$$
\sup \left|\frac{\partial^{2} S}{\partial y_{i} \partial y_{j}}(y)\right| \leqq \rho_{0}^{n} e^{-m\left(1-\eta_{k}\right)\left(1-\beta_{k}\right)|i-j|},
$$

for some constant B. As usual the factors of $\rho_{0}^{n}$ are chosen to keep the dimensions of the two sides equal.

Now define the change of variables by

$$
I_{i}=I_{i}^{\prime}+\frac{\partial S}{\partial \phi_{i}}\left(I^{\prime}, \phi\right), \quad \phi_{i}^{\prime}=\phi_{i}+\frac{\partial S}{\partial I_{i}^{\prime}}\left(I^{\prime}, \phi\right) .
$$

By the implicit function theorem of Appendix A, the first of the pair of Eqs. (3.8) may be inverted in the form:

$$
I^{\prime}=I+\Xi^{\prime}(I, \phi),
$$

for $\Xi^{\prime}(I, \phi)$ analytic on $W\left(r_{k}^{3}, \xi^{k}-\delta-1 ;\left\{I_{k}\right\}\right)$, provided

and

$$
\sup \sum_{i=1}^{N}\left|\frac{\partial^{2} S}{\partial I_{j}^{\prime} \partial \phi_{i}}\left(I^{\prime}, \phi\right)\right| \leqq(1 / 2)
$$

$$
\sup \left|\frac{\partial S}{\partial \phi_{j}}\right|<c_{1} \rho_{k+1} / k_{0},
$$

for all $j$, where the supremum runs over $W\left(r_{k}^{2}, \xi_{k}-\delta-1 ;\left\{I_{k}\right\}\right)$. Similarly the second equation in (3.8) is inverted in the form:

$$
\phi=\phi^{\prime}+\Delta\left(I^{\prime}, \phi^{\prime}\right)
$$

with $\Delta\left(I^{\prime}, \phi^{\prime}\right)$ analytic on $W\left(r_{k}^{2}, \xi_{k}-\delta-2 ;\left\{I_{k}\right\}\right)$, provided

and

$$
\sup \sum_{i=1}^{N}\left|\frac{\partial^{2} S}{\partial I_{i}^{\prime} \partial \phi_{j}}\left(I^{\prime}, \phi\right)\right| \leqq(1 / 2)
$$

$$
\sup \left|\frac{\partial S}{\partial I_{j}^{\prime}}\left(I^{\prime}, \phi\right)\right| \leqq(1 / 2)
$$

on $W\left(r_{k}^{2}, \xi_{k}-\delta-1 ;\left\{I_{k}\right\}\right)$ for all $j$. Both (3.10) and (3.12) follow from (3.7). Note that $\Xi^{\prime}(I, \phi)=-(\partial S / \partial \phi)\left(I^{\prime}, \phi\right)$ and $\Delta\left(I^{\prime}, \phi^{\prime}\right)=-\left(\partial S / \partial I^{\prime}\right)\left(I^{\prime}, \phi\right)$, so bounds on derivatives of $S$ lead to bounds on $\Xi^{\prime}$ and $\Delta$. We also define

$$
\phi^{\prime}=\phi+\Delta^{\prime}(I, \phi), \quad I=I^{\prime}+\Xi\left(I^{\prime}, \phi^{\prime}\right)
$$


where $\Delta^{\prime}(I, \phi)=(\partial S / \partial \phi)\left(I+\Xi^{\prime}(I, \phi), \phi\right)$ is defined and analytic on $W\left(r_{k}^{3}, \xi_{k}-\delta-1\right.$; $\left.\left\{I_{k}\right\}\right)$ and $\Xi\left(I^{\prime}, \phi^{\prime}\right)=(\partial S / \partial \phi)\left(I^{\prime}, \phi^{\prime}+\Delta\left(I^{\prime}, \phi^{\prime}\right)\right)$ is defined and analytic on $W\left(r_{k}^{2}, \xi_{k}-\right.$ $\left.\delta-2 ;\left\{I_{k}\right\}\right)$. Define the transformations

and

$$
C^{k}:\left(I^{\prime}, \phi^{\prime}\right) \rightarrow\left\{\begin{array}{l}
I=I^{\prime}+\Xi\left(I^{\prime}, \phi^{\prime}\right) \\
\phi=\phi^{\prime}+\Delta\left(I^{\prime}, \phi^{\prime}\right)
\end{array}\right.
$$

$$
\tilde{C}^{k}:(I, \phi) \rightarrow\left\{\begin{array}{l}
I^{\prime}=I+\Xi^{\prime}(I, \phi) \\
\phi^{\prime}=\phi+\Delta^{\prime}(I, \phi) .
\end{array}\right.
$$

Both are defined on $W\left(r_{k}^{3}, \xi_{k}-\delta-2 ;\left\{I_{k}\right\}\right)$ and map this set into $W\left(r_{k}^{2}, \xi_{k}-\delta-1\right.$; $\left\{I_{k}\right\}$ ), and on their common domain of definition $C^{k} \circ \widetilde{C}^{k}=\widetilde{C}^{k} \circ C^{k}=$ identity. Also, by construction, $C^{k}$ and $\widetilde{C}^{k}$ are canonical on $\operatorname{pr}_{1}\left(W\left(r_{k}^{3}, \xi_{k}-\delta-2 ;\left\{I_{k}\right\}\right)\right) \times T^{N}$. Define $\left(I_{k+1}, \phi_{k+1}\right)=\widetilde{C}^{k}\left(I_{k}, \phi_{k}\right)$. Since $\Xi^{\prime}(I, \phi)=-(\partial S / \partial \phi)\left(I^{\prime}(I, \phi), \phi\right)$, the bounds of Proposition 3.2 imply

$$
\left|\left(I_{k+1}\right)_{j}-\left(I_{k}\right)_{j}\right| \leqq \rho_{0}\left(\varepsilon_{k} \rho_{k}^{-1}\right)^{7 / 8} \text { for } j=1, \ldots, N .
$$

Thus, $W\left(r_{k}^{4}, \xi_{k}-\delta-2 ;\left\{I_{k+1}\right\}\right) \subset W\left(r_{k}^{3}, \xi_{k}-\delta-2 ;\left\{I_{k}\right\}\right)$, so $C^{k}$ and $\tilde{C}^{k}$ map $W\left(r_{k}^{4}\right.$, $\left.\xi_{k}-\delta-2 ;\left\{I_{k+1}\right\}\right) \rightarrow W\left(r_{k}^{2}, \xi_{k}-\delta-1 ;\left\{I_{k}\right\}\right) \subset W\left(\rho^{k}, \xi_{k},\left\{I_{k}\right\}\right)$. Define

$$
\begin{aligned}
H^{k+1}\left(I^{\prime}, \phi^{\prime}\right)= & H^{k} \circ C^{k}\left(I^{\prime}, \phi^{\prime}\right)=h^{k}\left(I^{\prime}+\Xi\left(I^{\prime}, \phi^{\prime}\right)\right) \\
& +f^{k, \mathrm{r}}\left(I^{\prime}+\Xi\left(I^{\prime}, \phi^{\prime}\right), \phi^{\prime}+\Delta\left(I^{\prime}, \phi^{\prime}\right)\right) \\
& +f^{k, \mathrm{nr}}\left(I^{\prime}+\Xi\left(I^{\prime}, \phi^{\prime}\right), \phi^{\prime}+\Delta\left(I^{\prime}, \phi^{\prime}\right)\right) .
\end{aligned}
$$

Define $f^{k[\leqq]}(I, \phi)=\sum_{\substack{v \in X_{k} \cup\{0\} \\ v \neq R^{k} \cup R_{p}^{k}}} f_{v}^{k}(I) e^{i v \cdot \phi}$ and define $f^{k[\geqq]}(I, \phi)=\sum_{\substack{v \notin X_{k} \\ v \notin R^{k} \cup R_{p}^{k} \\ v \neq 0}} f_{v}^{k}\left(I^{\prime}\right) e^{i v \cdot \phi}$. Then applying the fundamental theorem of calculus in a manner similar to $[3,4,6]$ we find

$$
\begin{aligned}
H^{k+1}\left(I^{\prime}, \phi^{\prime}\right)= & h^{k}\left(I^{\prime}\right)+f_{0}^{k}\left(I^{\prime}\right)+f^{k, \mathrm{r}}\left(I^{\prime}+\Xi\left(I^{\prime}, \phi^{\prime}\right), \phi^{\prime}+\Delta\left(I^{\prime}, \phi^{\prime}\right)\right) \\
& +f^{\mathrm{I}}\left(I^{\prime}, \phi^{\prime}\right)+f^{\mathrm{II}}\left(I^{\prime}, \phi^{\prime}\right)+f^{\mathrm{III}}\left(I^{\prime}, \phi^{\prime}\right)+f^{\mathrm{IV}}\left(I^{\prime}, \phi^{\prime}\right),
\end{aligned}
$$

with

$$
\begin{aligned}
f^{\mathrm{I}}\left(I^{\prime}, \phi^{\prime}\right) & =\int_{0}^{1} d t \int_{0}^{t} d s \frac{\partial^{2} h^{k}}{\partial I \partial I}\left(I^{\prime}+s \Xi\left(I^{\prime}, \phi^{\prime}\right)\right) \cdot \Xi\left(I^{\prime}, \phi^{\prime}\right) \cdot \Xi\left(I^{\prime}, \phi^{\prime}\right), \\
f^{\mathrm{II}}\left(I^{\prime}, \phi^{\prime}\right) & =\int_{0}^{1} d s \frac{\left.\partial f^{k[} \leqq\right]}{\partial I}\left(I^{\prime}+s \Xi\left(I^{\prime}, \phi^{\prime}\right), \phi^{\prime}+\Delta\left(I^{\prime}, \phi^{\prime}\right)\right) \cdot \Xi\left(I^{\prime}, \phi^{\prime}\right), \\
f^{\mathrm{III}}\left(I^{\prime}, \phi^{\prime}\right) & =f^{k[\geqq]}\left(I^{\prime}+\Xi\left(I^{\prime}, \phi^{\prime}\right), \phi^{\prime}+\Delta\left(I^{\prime}, \phi^{\prime}\right)\right) \text { and } \\
f^{\mathrm{IV}}\left(I^{\prime}, \phi^{\prime}\right) & =\sum_{v \in R_{p}^{k}} f_{v}^{k}\left(I^{\prime}+\Xi\left(I^{\prime}, \phi^{\prime}\right)\right) e^{i v \cdot \phi\left(I^{\prime}, \phi^{\prime}\right)} .
\end{aligned}
$$

In deriving (3.17) we used the fact that

$$
\frac{\partial h^{k}}{\partial I}\left(I^{\prime}\right) \cdot \Xi\left(I^{\prime}, \phi^{\prime}\right)+\sum_{\substack{v \in \mathbb{X}_{k} \\ v \notin \bar{R}^{k} \cup R_{p}^{k}}} f_{v}^{k}\left(I^{\prime}\right) e^{i v \cdot \phi\left(I^{\prime}, \phi^{\prime}\right)}=0,
$$


because of our definition of $S$. Setting $h^{k+1}\left(I^{\prime}\right)=h^{k}\left(I^{\prime}\right)+f_{0}^{k}\left(I^{\prime}\right)$ we see that

$$
\frac{\partial^{2} h^{k+1}}{\partial I_{i}^{\prime} \partial I_{j}^{\prime}}\left(I^{\prime}\right)=\delta_{i j}+\chi^{k}\left(I^{\prime} ; i, j\right)+\frac{\partial^{2} f_{0}^{k}}{\partial I_{i}^{\prime} \partial I_{j}^{\prime}}\left(I^{\prime}\right) \equiv \delta_{i j}+\chi^{k+1}\left(I^{\prime} ; i, j\right) .
$$

The bound (2.5) and a dimensional estimate implies

$$
\left|\frac{\partial^{2} f_{0}^{k}}{\partial I_{i}^{\prime} \partial I_{j}^{\prime}}\left(I^{\prime}\right)\right| \leqq B k_{0} \varepsilon_{k} \rho_{k+1}^{-1} \text { on } W\left(r_{k}^{1}, \xi_{k} ;\left\{I_{k}\right\}\right),
$$

while (2.10) implies

$$
\left|\frac{\partial^{2} f_{0}^{k}}{\partial I_{i}^{\prime} \partial I_{j}^{\prime}}\left(I^{\prime}\right)\right| \leqq e^{-\left(1-\eta_{k}\right) m|i-j|} \quad \text { for } \quad i \neq j,
$$

on the same domain. Combining these estimates with the bound on $\chi^{k}\left(I^{\prime} ; i, j\right)$ in $(2.9)$ we see that

$$
\sup \left|\chi^{k+1}\left(I^{\prime} ; i, j\right)\right| \leqq\left\{\begin{array}{lll}
c_{1}+B k_{0} \sum_{j=0}^{k-1} \varepsilon_{j} \rho_{j+1}^{-1}+B k_{0} \varepsilon_{k} \rho_{k+1}^{-1} & \text { if } & i=j \\
2 e^{-\left(1-\eta_{k}\right)|i-j|} \leqq e^{-\left(1-\eta_{k}-1\right) m|-j|} & \text { if } & i \neq j .
\end{array}\right.
$$

Thus, (2.9) can be iterated.

In the next section we begin the task of verifying that the bounds on the interaction terms can also be iterated.

\section{Some Preliminary Decay Estimates}

We begin iterating the estimates (2.5)-(2.7) and (2.10), which control the interaction terms in $H^{k+1}$. In this section we prove a series of estimates on various components of $f^{k+1}$. The first lemma is an application of the chain rule.

Lemma 4.1. Suppose $g$ is analytic on some domain $\mathscr{D} \subset \mathbb{C}^{2 N}$. We let $x_{j}$ represent either the $j^{\text {th }}$ or the $(j+N)^{\text {th }}$ coordinate of a point in $\mathbb{C}^{2 N}$. (This is in keeping with our notation in (1.14).) Suppose that

$$
\sup _{\mathscr{D}}\left|\frac{\partial g}{\partial x_{n}}(x)\right| \leqq C_{n}, \quad \sup _{\mathscr{D}}\left|\frac{\partial^{2} g}{\partial x_{n} \partial x_{p}}(x)\right| \leqq C_{n p}^{g} e^{-\kappa|n-p|}
$$

for some non-negative constants, $C_{n} C_{n p}^{g}$, and $\kappa(n, p=1, \ldots, N)$. Suppose $\tilde{x}$ is a holomorphic map from $\mathscr{D}^{\prime} \rightarrow \mathscr{D}$ satisfying

$$
\begin{aligned}
& \sup _{\mathscr{D}}\left|\frac{\partial \tilde{x}_{n}}{\partial x_{p}^{\prime}}\left(x^{\prime}\right)\right| \leqq C_{n p}^{1} e^{-\kappa|n-p|}, \\
& \sup _{\mathscr{D}^{\prime}}\left|\frac{\partial^{2} \tilde{x}_{m}}{\partial x_{n}^{\prime} \partial x_{p}^{\prime}}\left(x^{\prime}\right)\right| \leqq C_{m n p}^{2} e^{-\kappa|n-p|},
\end{aligned}
$$

for some constants $C_{n p}^{1}$ and $C_{m n p}^{2}$. Here, in analogy with our notation above, we let $\tilde{x}_{j}$ denote either the $j^{\text {th }}$ or the $(j+N)^{\text {th }}$ component of the map. (See Lemma 4.2 for an 
explicit example of this notation.) Then

$$
\sup _{\mathscr{D}^{\prime}}\left|\frac{\partial}{\partial x_{i}^{\prime}}\left(g \circ \tilde{x}\left(x^{\prime}\right)\right)\right| \leqq D_{i}, \quad \text { and } \sup _{\mathscr{D}^{\prime}}\left|\frac{\partial^{2}}{\partial x_{i}^{\prime} \partial x_{j}^{\prime}}\left(g \circ \tilde{x}\left(x^{\prime}\right)\right)\right| \leqq D e^{-\kappa|i-j|} .
$$

Here $D_{i}=2 \sum_{m=1}^{N} C_{m} C_{m i}^{1}$ and $D=4 \sup _{i j}\left(\sum_{n=1}^{N} C_{n} C_{n i j}^{2}+\sum_{m, n=1}^{N} C_{m n}^{g} C_{m i}^{1} C_{n j}^{1}\right)$. The easy proof is omitted. Note that (4.3) can always be satisfied by picking $D_{i}$ and $D$ sufficiently large. The point of the lemma is that $D_{i}$ and $D$ may be chosen to have the stated form.

The next lemma tells how the changes of variables $I_{i}=I_{i}\left(I^{\prime}, \phi^{\prime}\right)$ and $\phi_{i}=\phi_{i}\left(I^{\prime}, \phi^{\prime}\right)$ depend on the variables $I_{j}^{\prime}$ and $\phi_{i}^{\prime}$ as $|i-j|$ becomes large. To state the result concisely, let $x_{i}^{\prime}$ be either $I_{i}^{\prime}$ or $\phi_{i}^{\prime}$, and let $\tilde{x}_{i}\left(x^{\prime}\right)$ be either $I_{i}\left(I^{\prime}, \phi^{\prime}\right)$ or $\phi_{i}\left(I^{\prime}, \phi^{\prime}\right)$ depending on the context.

Lemma 4.2. Let $\mathscr{D}^{\prime}=W\left(r_{k}^{4}, \xi_{k}-\delta-3 ;\left\{I_{k}\right\}\right)$. Then

$$
\begin{aligned}
& \sup _{\mathscr{D}^{\prime}}\left|\frac{\partial \tilde{x}_{i}}{\partial x_{j}^{\prime}}\left(x^{\prime}\right)-\tilde{\delta}_{i j}\right| \\
& \quad \leqq \rho_{0}^{n} \min \left(\left(\lambda\left(\varepsilon_{0}\right)\right)^{-2} k_{0} \varepsilon_{k} \rho_{k+1}^{-1} B^{L_{k}}, e^{-m\left(1-\eta_{k}\right)\left(1-3 \beta_{k}\right)|i-J|}\right) .
\end{aligned}
$$

Also

$$
\sup _{\mathscr{D}^{\prime}}\left|\frac{\partial^{2} \tilde{x}_{\ell}}{\partial x_{i}^{\prime} \partial x_{j}^{\prime}}\left(x^{\prime}\right)\right| \leqq B \rho_{0}^{n}\left[\left(r_{k}^{2}\right)_{\ell}-\left(r_{k}^{3}\right)_{\ell}\right]^{-1} \times e^{-m\left(1-\eta_{k}\right)\left(1-4 \beta_{k}\right)|i-j|}
$$

Here, $\tilde{\delta}_{i j}=1$ if $\tilde{x}_{i}=I_{i}\left(I^{\prime}, \phi^{\prime}\right)$ and $x_{i}^{\prime}=I_{i}^{\prime}$ or $\tilde{x}_{i}=\phi_{i}\left(I^{\prime}, \phi^{\prime}\right)$ and $x_{i}^{\prime}=\phi_{i}^{\prime}$, and $\tilde{\delta}_{i j}=0$ otherwise. This slightly awkward definition is necessitated by the fact that if $\tilde{x}_{i}=I_{i}\left(I^{\prime}, \phi^{\prime}\right)$ and $x_{i}^{\prime}=\phi_{i}^{\prime}\left(\right.$ or if $\tilde{x}_{i}=\phi_{i}\left(I^{\prime}, \phi^{\prime}\right)$ and $\left.x_{i}^{\prime}=I_{i}^{\prime}\right)$, then $\left|\frac{\partial \tilde{x}_{i}}{\partial x_{i}^{\prime}}\right| \ll 1-\operatorname{not} \mathcal{O}(1)$. However, this notation has proved so convenient otherwise that I think it is worth putting up with this problem. The constant $n$ is chosen as usual to insure that the dimensions of the two sides are equal.

Remark. Note that $\left(r_{k}^{2}\right)_{\ell}-\left(r_{k}^{3}\right)_{\ell} \sim \mathcal{O}\left(\left(b^{m}\right)_{\ell}\right)$ if $\ell \in S^{m}$ for some $m=0, \ldots, k-2$, and $\left(r_{k}^{2}\right)_{\ell}-\left(r_{k}^{3}\right)_{\ell} \sim \mathcal{O}\left(\rho_{k+1}\right)$ otherwise, so the "size" of these derivatives depends on the order of the site $\ell$.

At numerous points in what follows we will have occasion to bound sums over $v \in \mathbb{Z}^{N}$, in which the summands obey certain estimates that we wish to show are inherited by the sum. Estimates on the sums are provided by

Proposition 4.3. Suppose $g(x)=\sum_{v \in \mathbb{Z}^{N}} g_{v}(x)$ and

$$
\sup _{\mathscr{D}}\left|\frac{\partial g_{v}}{\partial x_{i}}(x)\right| \leqq \min \left\{\begin{array}{l}
K_{1} e(i) e^{-\delta^{\prime}|v|} \\
K_{1} e^{-\kappa_{1}|i-\ell|} e^{-\delta^{\prime}|v|}
\end{array}\right.
$$

for some domain $\mathscr{D}$, some constant $e(i)$ and $\ell$ the point in $\operatorname{supp} v$ such $|\ell-i|$ is 
maximized. Then if $\delta^{\prime}$ and $\kappa_{1}$ are greater than 6 ,

$$
\sup _{\mathscr{D}}\left|\frac{\partial g}{\partial x_{i}}(x)\right| \leqq K_{1} e(i) B^{L}
$$

where $L$ is any number greater than $\left(2 / \kappa_{1}\right)|\ln e(i)|$, and $B$ is some universal constant.

Suppose further that

$$
\sup _{\mathscr{D}}\left|\frac{\partial^{2} g_{v}}{\partial x_{i} \partial x_{j}}(x)\right| \leqq\left\{\begin{array}{l}
K_{2} e^{-\kappa_{2}|i-j|} e^{-\delta^{\prime}|v|} \\
K_{3} e^{-\kappa_{2}|i-\ell|} e^{-\delta^{\prime}|v|}
\end{array}\right.
$$

and $\kappa_{2}>6$. Then

$$
\sup _{\mathscr{D}}\left|\frac{\partial^{2} g}{\partial x_{i}^{\prime} \partial x_{j}^{\prime}}(x)\right| \leqq\left(K_{2}+K_{3}\right) e^{-\kappa_{2}|i-j|} B^{(i-j \mid+1)}
$$

for some constant $B$.

The proofs of Lemma 4.2 and Proposition 4.3 are presented in Sect. 7. With these two results we are ready to study $H^{k+1}$, beginning with these corollaries of Proposition 4.3.

Corollary 4.4. Let $\mathscr{D}=W\left(r_{k}^{1}, \xi_{k}-\delta ;\left\{I_{k}\right\}\right)$. Then

$$
\sup _{\mathscr{D}}\left\{\left|\frac{\partial f^{k[\geqq]}}{\partial I_{i}}\right|+\rho_{k+1}^{-1}\left|\frac{\partial f^{k[\geqq]}}{\partial \phi_{i}}\right|\right\} \leqq \rho_{0}^{2} k_{0} \rho_{k}^{-1} B^{L_{k+1}}\left(\varepsilon_{0} \rho_{0}^{-1}\right)^{(13 / 8)(3 / 2)^{k}},
$$

for $i=1, \ldots, N$. Let $x_{i}$ be as in (1.14), then

$$
\left|\frac{\partial^{2} f^{k[\geqq]}}{\partial x_{i} \partial x_{j}}(x)\right| \leqq B \rho_{0}^{n} e^{-m\left(1-\eta_{k}\right)\left(1-3 \beta_{k}\right)(i-j)},
$$

on $W\left(r_{k}^{2}, \xi_{k}-\delta-1 ;\left\{I_{k}\right\}\right)$, where as usual the factors of $\rho_{0}$ are chosen to keep the dimensions of the two sides the same.

Proof.

$$
\frac{\partial f^{k[\geqq]}}{\partial x_{i}}(x)=\sum_{\substack{v \notin x_{k} \\ v \neq 0}} \frac{\partial}{\partial x_{i}}\left(g_{v}(x)\right),
$$

where $g_{v}(x)=f_{v}^{k}(I) e^{i v \cdot \phi}$. Using (2.5), (2.10), the inductive hypotheses, and Cauchy's theorem bound $\left|\left(\partial / \partial I_{i}\right)\left(f_{v}^{k}(I) e^{i v \cdot \phi}\right)\right|$ by $\min \left(B k_{0} \rho_{k+1}^{-1} \rho_{0}^{2} e^{-m\left(1-\eta_{k}\right) d(s u p p v)}\right.$ $\left.e^{-\delta|v|}, \rho_{0} e^{-m\left(1-\eta_{k}|i-\ell|\right.} e^{-\delta|v|}, \varepsilon_{k} e^{-\delta|v|}\right)$ on $\mathscr{D}$, which is in turn bounded by min $\left(\rho_{0}\left(\varepsilon_{0} \rho_{0}^{-1}\right)^{(13 / 8)(3 / 2)^{k}} e^{-\delta|v| / 4}, \rho_{0} e^{-m\left(1-\eta_{k}|i-\ell|\right.} e^{-\delta|v|}\right)$, since $v \notin \mathbb{X}_{k}$. (Recall that $\ell$ is the point in $\operatorname{supp} v$ furthest from $i$ ). In deriving this estimate we have used a little trick that we will often call on below, and so we mention it here. If $j$ and $\ell$ are the two most widely separated sites in $\operatorname{supp} v$, then by integrating by parts,

$$
f_{v}^{k}(I)=\int d \phi\left(-\frac{1}{v_{j} v_{\ell}}\right)\left[\frac{\partial^{2}}{\partial \phi_{j} \partial \phi_{\ell}} f^{k}(I, \phi)\right] e^{i v \cdot \phi},
$$

where $\int d \phi \equiv\left(\prod_{i=1}^{N} \int_{0}^{2 \pi}\left(d \phi_{i} / 2 \pi\right)\right)$. Thus $\partial f_{v}^{k} / \partial I_{i}$ is the $v^{\text {th }}$ harmonic of the function 
$-\left(1 / v_{j} v_{\ell}\right)\left[\left(\partial^{3} / \partial I_{i} \partial \phi_{j} \partial \phi_{\ell}\right) f^{k}(I, \phi)\right]$, which with the aid of $(2.10)$ and a dimensional estimate we bound in magnitude by $B \rho_{0}^{n} k_{0} \rho_{k+1}^{-1} e^{-\left(1-\eta_{k}\right) m|\ell-j|}$. But $|\ell-j|=d(\operatorname{supp} v)$, and the first estimate above follows from Cauchy's theorem. Similarly $\left(\partial / \partial \phi_{i}\right)\left(g_{v}(x)\right)=f_{v}(I)\left(i v_{i}\right) e^{i v \cdot \phi}=0$ unless $i \in \operatorname{supp} v$. Using this observation, plus (2.5), (2.10) and Cauchy's theorem, we find $\left|\left(\partial / \partial \phi_{i}\right) g_{v}(x)\right|$ is bounded by

$$
\begin{aligned}
& \min \left(\rho_{0} \varepsilon_{k}|v| e^{-\delta|v|}, \rho_{0}^{2} e^{-m\left(1-\eta_{k}\right)|i-\ell|}|v| e^{-\delta|v|}, B \rho_{0}^{2} e^{-m\left(1-\eta_{k}\right) d(\text { supp } v)}|v| e^{-\delta|v|}\right) \\
& \quad \leqq \min \left(\rho_{0}^{2}\left(\varepsilon_{0} \rho_{0}^{-1}\right)^{(13 / 8)(3 / 2)^{k}} e^{-\delta|v| / 4}, \rho_{0}^{2} e^{-m\left(1-\eta_{k}\right)|i-\ell|} e^{-\delta|v| / 2}\right) .
\end{aligned}
$$

Applying (4.7) yields (4.10). Next note that (2.5), (2.10) and Cauchy's theorem imply

$$
\begin{aligned}
\left|\frac{\partial^{2} g_{v}}{\partial x_{i} \partial x_{j}}(x)\right| & \leqq \rho_{0}^{n} \min \left\{\begin{array}{l}
\varepsilon_{k}\left[\left(\rho^{k}\right)_{j}-\left(r_{k}^{1}\right)_{j}\right]^{-1} e^{-\delta|v|} \\
e^{-m\left(1-\eta_{k}\right)|i-\jmath|} e^{-\delta|v|} \\
\rho_{0}\left[\left(\rho^{k}\right)_{j}-\left(r_{k}^{1}\right)_{j}\right]^{-1} e^{-m\left(1-\eta_{k}\right)|t-\ell|} e^{-\delta|v|}
\end{array}\right. \\
& \leqq \rho_{0}^{n} \min \left\{\begin{array}{l}
e^{-m\left(1-\eta_{k}\right)|t-\jmath|} e^{-\delta|v|} \\
e^{-m\left(1-\eta_{k}\right)\left(1-2 \beta_{k}\right)|i-j|} e^{-m \beta_{k}\left(1-\eta_{k}\right)|i-\ell|} e^{-\delta|v|},
\end{array}\right.
\end{aligned}
$$

where as usual $n$ is chosen to keep the dimensions correct. In addition the last inequality used the fact that if $\chi<\min \left(c_{1}, c_{2}\right)$, one has $\chi<c_{1}^{\beta} c_{2}^{(1-\beta)}$ for $\beta \in[0,1]$. Inequality (4.11) then follows from the second half of Proposition 4.3. (Note that we can assume $|i-j|>(1 / 8) L_{k}$, since otherwise (4.11) follows from (4.10) by a dimensional estimate.)

In like fashion, if we let $f^{4}(I, \phi)=\sum_{v \in R_{k}^{p}} f_{v}(I) e^{i v \phi}$, we have

Corollary 4.5. If $\mathscr{D}=W\left(r_{k}^{1}, \xi_{k}-\delta\right.$; $\left.\left\{I_{k}\right\}\right)$, then

$$
\begin{array}{r}
\sup _{\mathscr{D}}\left\{\left|\frac{\partial f^{k[\leqq]}}{\partial I_{i}}\right|+\rho_{0}^{-1}\left|\frac{\partial f^{k[\leqq]}}{\partial \phi_{i}}\right|\right\} \leqq \varepsilon_{k} B^{L_{k}}, \\
\sup _{\mathscr{D}}\left\{\left|\frac{\partial f^{4}}{\partial I_{i}}\right|+\rho_{0}^{-1}\left|\frac{\partial f^{4}}{\partial \phi_{i}}\right|\right\} \leqq \varepsilon_{k} B^{L_{k}},
\end{array}
$$

and

$$
\sup _{\mathscr{D}}\left\{\left|\frac{\partial f^{k, \mathrm{r}}}{\partial I_{i}}\right|+\rho_{0}^{-1}\left|\frac{\partial f^{k, \mathrm{r}}}{\partial \phi_{i}}\right|\right\} \leqq A(k, i) B^{\tilde{\mathcal{L}}(i)},
$$

where $\tilde{L}(i)=L_{m}$ if $i \in S^{m}, m=0, \ldots, k-1, \tilde{L}(i)=L_{k}$ otherwise. Also, if $x$ is as in (1.14), then on $W\left(r_{k}^{2}, \xi_{k}-\delta-1 ;\left\{I_{k}\right\}\right)$,

and

$$
\begin{aligned}
& \sup \left|\frac{\partial^{2} f^{k[\leqq]}}{\partial x_{i} \partial x_{j}}(x)\right| \leqq \rho_{0}^{n} e^{-m\left(1-\eta_{k}\right)\left(1-2 \beta_{k}\right)(i-j \mid}, \\
& \sup \left|\frac{\partial^{2} f^{4}}{\partial x_{i} \partial x_{j}}(x)\right| \leqq \rho_{0}^{n} e^{-m\left(1-\eta_{k}\right)\left(1-2 \beta_{k}\right)|i-j|},
\end{aligned}
$$

$$
\sup \left|\frac{\partial^{2} f^{k, \mathrm{r}}}{\partial x_{i} \partial x_{j}}(x)\right| \leqq \rho_{0}^{n} e^{-m\left(1-\eta_{k}\right)\left(1-2 \beta_{k}\right)|i-j|}
$$


Once again the factors of $\rho_{0}$ are chosen to "fix" the dimensions.

Proof. By (2.5), (2.6), (2.10), and Cauchy's theorem we have

$$
\left|\frac{\partial f_{v}^{k}}{\partial I_{j}} e^{i v \phi}\right|+\rho_{0}^{-1}\left|f_{v}^{k}\left(v_{j}\right) e^{i v \cdot \phi}\right| \leqq \min \left(A(k, j)|v| e^{-\delta|v|}, \rho_{0} e^{-m\left(1-\eta_{k}\right)|j-\ell|}|v| e^{-\delta|v|}\right),
$$

while

$$
\begin{aligned}
\left|\frac{\partial^{2} f_{v}}{\partial x_{i} \partial x_{j}}(x)\right| \leqq & \rho_{0}^{n} \min \left(e^{-m\left(1-\eta_{k}\right)|i-j|} e^{-\delta|v|}, A(k, i)\right. \\
& \left.\cdot\left[\left(\rho^{k}\right)_{j}-\left(r_{k}^{1}\right)_{j}\right]^{-1} e^{-\delta|v|}, \rho_{0}\left[\left(\rho^{k}\right)_{j}-\left(r_{k}^{1}\right)_{j}\right]^{-1} e^{-|i-\ell|} e^{-\delta|v|}\right),
\end{aligned}
$$

on $W\left(r_{k}^{1}, \xi_{k}-\delta ;\left\{I_{k}\right\}\right)$ with $\ell$ the point in $\operatorname{supp} v$ such that $|i-\ell|$ is maximized. Note that this last inequality implies

$$
\begin{aligned}
\left|\frac{\partial^{2} f_{v}^{k}}{\partial x_{i} \partial x_{j}}(x)\right| \leqq & \rho_{0}^{n} \min \left(e^{-m\left(1-\eta_{k}\right)|i-j|} e^{-\delta|v|}\right. \\
& \left.\cdot e^{-m\left(1-\eta_{k}\right)\left(1-2 \beta_{k}\right)|\imath-j|} e^{-m\left(1-\eta_{k}\right) \beta_{k}|l-\ell|} e^{-\delta|v|}\right) .
\end{aligned}
$$

(The last step used the fact, easily derived from the definitions of the $m^{\text {th }}$ order zones, $S^{m}$, that if $i \in S^{m}$ and $L_{m+n} \leqq|i-j|<L_{m+n+1}$, then $\left|\left[\left(\rho^{k}\right)_{j}-\left(r_{k}^{1}\right)_{j}\right]^{-1}\right| \leqq B k_{0} \rho_{m+n+2}^{-1}$.) Proposition 4.3 then yields (4.15) and the last of the three inequalities in (4.14). (Note that we may assume $|i-j|>(1 / 8) L_{k}$, in the first two inequalities in (4.15), and $|i-j|>(1 / 8) \max (\widetilde{L}(i), \widetilde{L}(j))$ in the last of these inequalities since otherwise they follow immediately from (4.14) by dimensional estimates.) The first two inequalities of (4.14) follow if we note that all the Fourier coefficients $f_{v}^{k}(I)$ in $f^{k[\leqq]}$ and $f^{4}$ satisfy $\nu \notin \widetilde{R}$, so by (2.5) and Cauchy's theorem we have

$$
\sup \left\{\left|\frac{\partial f_{v}^{k}}{\partial I_{j}}(I) e^{i v \cdot \phi}\right|+\rho_{0}^{-1}\left|f_{v}^{k}(I)\left(v_{j}\right) e^{i v \cdot \phi}\right|\right\} \leqq \varepsilon_{k} e^{-\delta|v|}
$$

on $W\left(\rho^{k}, \xi_{k}-\delta\right.$; $\left.\left\{I_{k}\right\}\right)$ for these harmonics. Combining this estimate with those above, and then applying Proposition 4.3 completes the proof of (4.14).

\section{The Short Range Nature of $f^{k+1}$}

We demonstrate in this section that the estimate (2.10) holds for the Hamiltonian $H^{k+1}$. Let $\tilde{x}\left(x^{\prime}\right)$ be as in Lemma 4.2. If $\mathscr{D}^{\prime}=W\left(r_{k}^{4}, \xi_{k}-\delta-3 ;\left\{I_{k}\right\}\right)$ and $\mathscr{D}=$ $W\left(r_{k}^{3}, \xi_{k}-\delta-2 ;\left\{I_{k}\right\}\right)$, then $\tilde{x}: \mathscr{D}^{\prime} \rightarrow \mathscr{D}$. If $f^{\mathrm{III}}$ and $f^{\mathrm{IV}}$ are as in (3.17) we have $f^{\mathrm{III}}\left(I^{\prime}, \phi^{\prime}\right)=f^{k[\geq]} \circ \tilde{x}\left(x^{\prime}\right)$ and $f^{\mathrm{IV}}\left(I^{\prime}, \phi^{\prime}\right)=f^{4} \circ \tilde{x}\left(x^{\prime}\right)$, while $f^{k, r}\left(I^{\prime}+\Xi\left(I^{\prime}, \phi^{\prime}\right), \phi^{\prime}+\right.$ $\left.\Delta\left(I^{\prime}, \phi^{\prime}\right)\right)=f^{k, r_{\circ}} \tilde{x}\left(x^{\prime}\right)$. We then have

\section{Proposition 5.1.}

$$
\begin{aligned}
& \sup _{\mathscr{D}^{\prime}}\left|\frac{\partial f^{\mathrm{III}}}{\partial x_{i}^{\prime}}\left(x^{\prime}\right)\right| \leqq \rho_{0}^{n} \rho_{k+1}^{-1}\left(\varepsilon_{0} \rho_{0}^{-1}\right)^{(13 / 8)(3 / 8)^{k}} B^{L_{k}}, \\
& \sup _{\mathscr{D}^{\prime}}\left|\frac{\partial f^{\mathrm{IV}}}{\partial x_{i}^{\prime}}\left(x^{\prime}\right)\right| \leqq \rho_{0}^{n} \varepsilon_{k} B^{L_{k}},
\end{aligned}
$$


and

$$
\sup _{\mathscr{D}^{\prime}}\left|\frac{\partial f^{k, \mathrm{r}}}{\partial x_{i}^{\prime}} \circ \tilde{x}\left(x^{\prime}\right)\right| \leqq \rho_{0}^{n} A(k, i) B^{\tilde{L}(i)} .
$$

Furthermore on $W\left(r_{k}^{5}, \xi_{k}-\delta-4 ;\left\{I_{k}\right\}\right)$

$$
\begin{aligned}
& \left|\frac{\partial^{2} f^{\mathrm{III}}}{\partial x_{i}^{\prime} \partial x_{j}^{\prime}}\left(x^{\prime}\right)\right| \leqq\left(\rho_{0}^{n} / 2^{3}\right) e^{-m\left(1-\eta_{k+1}\right)|i-j|}, \\
& \left|\frac{\partial^{2} f^{\mathrm{III}}}{\partial x_{i}^{\prime} \partial x_{j}^{\prime}}\left(x^{\prime}\right)\right| \leqq\left(\rho_{0}^{n} / 2^{3}\right) e^{-m\left(1-\eta_{k+1}\right)|i-j|},
\end{aligned}
$$

and

$$
\left|\frac{\partial^{2} f^{k, \mathrm{r}}}{\partial x_{i}^{\prime} \partial x_{j}^{\prime}} \circ \tilde{x}\left(x^{\prime}\right)\right| \leqq\left(\rho_{0}^{n} / 2^{3}\right) e^{-m\left(1-\eta_{k+1}\right)|i-j|} .
$$

Proof. Apply Lemma 4.1, taking the function $g(x)$ in that lemma to be $f^{k[\geqq]}, f^{4}$, and $f^{k, \mathrm{r}}$ respectively in each of the inequalities in (5.1) and (5.2). Choose $\kappa$ (in (4.1) and (4.2)) equal to zero for (5.1). From Lemma 4.2 take $C_{i j}^{1}=2$ if $i=j$, and

$$
C_{i j}^{1}=\min \left(\rho_{0}^{n} e^{-m\left(1-\eta_{k}\right)\left(1-4 \beta_{k}\right)|i-j|}, \rho_{0}^{n}\left(\lambda\left(\varepsilon_{0}\right)\right)^{-2} k_{0} \varepsilon_{k} \rho_{k+1}^{-1} B^{L_{k}}\right) \text { if } i \neq j .
$$

From Corollaries 4.4 and 4.5 we see that the constants $C_{\ell}$ may be chosen to be $\rho_{0}^{n} B^{L_{k+1}}\left(\varepsilon_{0} \rho_{0}^{-1}\right)^{(13 / 8)(3 / 2)^{k}}, \rho_{0}^{n} \varepsilon_{k} B^{L_{k}}$ and $\rho_{0}^{n} A(k, \ell) B^{\tilde{\mathcal{L}(\ell)}}$ respectively in the cases $g$ equals $f^{k[\geqq]}, f^{4}$, and $f^{k, r}$. Inequalities (5.1) then follow from the first inequality in (4.3). In calculating the constant that appears on the right-hand side of the third inequality, we note that $k_{0} A(k, p)\left(\varepsilon_{k} \rho_{k+1}^{-1}\right) B^{L_{k}} \leqq A(k, i) B^{\mathcal{L}(i)}$ for all sites $p$, while $k_{0} \varepsilon_{k} \rho_{k+1}^{-1} B^{L_{k}}<$ $e^{-m\left(1-\eta_{k}\right)\left(1-4 \beta_{k}\right) i-p \mid / 2}$ for at most $B L_{k}$ sites $p$, so

$$
\begin{aligned}
\sum_{p} C_{p} C_{i p}^{1} & \leqq \\
& \leqq A\left(2+2^{2}\right) A(k, i) B^{\prime \prime} \mathcal{L}^{(i)} .
\end{aligned}
$$

For $(5.2)$ choose $\kappa=m\left(1-\eta_{k}\right)\left(1-5 \beta_{k}\right)$. Then $C_{i j}^{1}=2$ if $i=j$ and $C_{i j}^{1}=\rho_{0}^{n}$ $e^{-m\left(1-\eta_{k}\right) \beta_{k}|i-j|}$ if $i \neq j$ by Lemma 4.2 .

$$
C_{\ell i j}^{2}=B \rho_{0}^{n}\left[\left(r_{k}^{2}\right)_{\ell}-\left(r_{k}^{3}\right)_{\ell}\right]^{-1} e^{-m\left(1-\eta_{k}\right) \beta_{k}|i-j|} \quad \text { if } \quad|i-\ell|<2|i-j|
$$

and

$$
C_{\ell i j}^{2}=\rho_{0}^{n}\left[\left(r_{k}^{4}\right)_{j}-\left(r_{k}^{5}\right)_{j}\right]^{-1} e^{-m\left(1-\eta_{k}\right)\left(1-3 \beta_{k}\right) / \xi-\ell / / 2}
$$

otherwise. This estimate follows by noting that (4.4) and a dimensional estimate on $W\left(r_{k}^{5}, \xi_{k}-\delta-4 ;\left\{I_{k}\right\}\right)$ imply

$$
\begin{aligned}
\left|\frac{\partial^{2} \tilde{x}}{\partial x_{i}^{\prime} \partial x_{j}^{\prime}}\right| & \leqq B \rho_{0}^{n}\left[\left(r_{k}^{4}\right)_{j}-\left(r_{k}^{5}\right)_{j}\right]^{-1} e^{-m\left(1-\eta_{k}\right)\left(1-3 \beta_{k}\right)|i-\ell|} \\
& \leqq B \rho_{0}^{n}\left[\left(r_{k}^{4}\right)_{j}-\left(r_{k}^{5}\right)_{j}\right]^{-1} e^{-m\left(1-\eta_{k}\right)\left(1-3 \beta_{k}\right)|i-\ell| / 2} e^{-m\left(1-\eta_{k}\right)\left(1-3 \beta_{k}\right)_{i}-\jmath \mid}
\end{aligned}
$$

if $|i-\ell|>2|i-j|$. 
Choose the constants $C_{n}$, as we did in the previous paragraph. Corollaries 4.4 and 4.5 also imply that the constants $C_{m n}^{g}$ may be chosen to be $\rho_{0}^{n} e^{-m\left(1-\eta_{k}\right)|m-n|}$ in each of the three cases. To prove (5.2) first note that we may assume $|i-j|>(1 / 8) L_{k}$ in the first two inequalities in (5.2) while in the third inequality we can assume $|i-j|>$ $(1 / 8) \max (\tilde{L}(i), \tilde{L}(j))$, since (5.2) would follow from (5.1) and a dimensional estimate were this not the case. Given this assumption, (5.2) follows from the second inequality in (4.3), since a straightforward computation of the constant $D$ appearing there shows it may be bounded by $\left(\rho_{0}^{n} / 2^{3}\right)$ in each case.

We now study the two remaining terms in (3.17), $f^{\mathrm{I}}$ and $f^{\mathrm{II}}$. We first prove the following easy consequence of the product rule

Lemma 5.2. Suppose $f_{\ell}$ and $g_{\ell}(\ell=1, \ldots, N)$ are analytic on some domain $\mathscr{D} \subset \mathbb{C}^{2 N}$, and satisfy the bounds

$$
\begin{aligned}
\sup \left|g_{\ell}\right| & <C_{g \ell}, \sup \left|f_{\ell}\right|<C_{f \ell}, \\
\sup \left|\frac{\partial g_{\ell}}{\partial x_{i}}\right| & <\min \left(C_{g \ell}^{1}, C_{g \ell}^{2} e^{-\kappa|i-\ell|}\right), \\
\sup \left|\frac{\partial f_{\ell}}{\partial x_{i}}\right| & <\min \left(C_{f \ell}^{1}, C_{f \ell}^{2} e^{-\kappa|i-\ell|}\right), \\
\sup \left|\frac{\partial^{2} g_{\ell}}{\partial x_{i} \partial x_{j}}\right| & <\min \left(C_{g \ell}^{3} e^{-\kappa|i-j|}, C_{g \ell}^{4} e^{-\kappa|i-\ell|}\right),
\end{aligned}
$$

and

$$
\sup \left|\frac{\partial^{2} f_{\ell}}{\partial x_{i} \partial x_{j}}\right|<\min \left(C_{f \ell}^{3} e^{-\kappa|i-\jmath|}, C_{f \ell}^{4} e^{-\kappa|i-\ell|}\right),
$$

where we again let $x_{j}$ represent either the $j^{\text {th }}$ or $(j+N)^{\text {th }}$ component of $x \in \mathbb{C}^{2 N}$. Then if $\kappa>0$, and $L$ is a nonnegative integer we have

$$
\begin{aligned}
\sup _{\mathscr{D}}\left|\frac{\partial}{\partial x_{i}}\left(\sum_{\ell=1}^{N} f_{\ell}(x) g_{\ell}(x)\right)\right| & \leqq 2^{2}\left(1-e^{-\kappa}\right)^{-1} \sup _{\ell}\left(C_{g \ell} C_{f \ell}^{2}+C_{f \ell} C_{g \ell}^{2}\right) e^{-\kappa L} \\
& +2^{2} \sup _{\ell}\left(C_{g \ell} C_{f \ell}^{1}+C_{g \ell}^{1} C_{f \ell}\right)(L) .
\end{aligned}
$$

Furthermore,

$$
\begin{aligned}
& \sup _{\mathscr{Q}}\left|\frac{\partial^{2}}{\partial x_{i} \partial x_{j}}\left(\sum_{\ell=1}^{N} f_{\ell}(x) g_{\ell}(x)\right)\right| \leqq\left[2\left(|i-j|+2\left(1-e^{-\kappa}\right)^{-1}\right) C_{f \ell}^{2} C_{g \ell}^{2}\right. \\
& \quad+\sup _{\ell}\left(2^{2}|i-j|+2^{2}\right)\left(C_{f \ell} C_{g \ell}^{3}+C_{g \ell} C_{f \ell}^{3}\right) \\
& \left.\quad+2^{2} \sup _{\ell}\left(C_{f \ell} C_{g \ell}^{4}+C_{g \ell} C_{f \ell}^{4}\right)\right]\left(1-e^{-\kappa}\right)^{-1} e^{-\kappa|\imath-\jmath|}
\end{aligned}
$$


Proof. By the product rule,

$$
\begin{aligned}
\left|\frac{\partial}{\partial x_{i}}\left(\sum_{\ell} f_{\ell}(x) g_{\ell}(x)\right)\right| \leqq & \sum_{\ell}\left|g_{\ell}(x) \frac{\partial f_{\ell}}{\partial x_{i}}(x)+f_{\ell}(x) \frac{\partial g_{\ell}}{\partial x_{i}}(x)\right| \\
\leqq & \sum_{\substack{\ell: \\
|i-\ell| \geqq L}}\left(C_{g \ell} C_{f \ell}^{2}+C_{f \ell} C_{g \ell}^{2}\right) e^{-\kappa|i-\ell|} \\
& +\sum_{\substack{\ell: \vdots \\
|i-\ell|<L}}\left(C_{g \ell} C_{f \ell}^{1}+C_{f \ell} C_{g \ell}^{1}\right)
\end{aligned}
$$

and (5.6) follows immediately.

Applying this product rule a second time we find

$$
\begin{aligned}
\left|\frac{\partial^{2}}{\partial x_{i} \partial x_{j}}\left(\sum f_{\ell}(x) g_{\ell}(x)\right)\right| \leqq & \sum_{\ell} \mid g_{\ell}(x) \frac{\partial^{2} f_{\ell}}{\partial x_{i} \partial x_{j}}(x)+f_{\ell}(x) \frac{\partial^{2} g_{\ell}}{\partial x_{i} \partial x_{j}}(x) \\
& +\frac{\partial g_{\ell}}{\partial x_{i}}(x) \frac{\partial f_{\ell}}{\partial x_{j}}(x)+\frac{\partial f_{\ell}}{\partial x_{i}}(x) \frac{\partial g_{\ell}}{\partial x_{j}}(x) \mid
\end{aligned}
$$

Bound the last two terms by bounding the derivatives by $C_{g \ell}^{2} C_{f \ell}^{2} e^{-\kappa[|i-\ell|+|\jmath-\ell|]}$ and then summing over $\ell$ to obtain the term $\sup \left(2(|i-j|)+2\left(1-e^{-\kappa}\right)^{-1}\right) C_{g \ell}^{2} C_{f \ell}^{2} e^{-\kappa|i-j|}$. Bound the second term by first summing over all $\ell$ such that $|i-\ell| \leqq 2|i-j|$. If we bound $f_{\ell}$ by $C_{f \ell}$ and bound $\left|\partial^{2} g_{\ell} / \partial x_{i} \partial x_{j}\right|$ by $C_{g \ell}^{3} e^{-\kappa|i-j|}$, these terms give a contribution of $\sup 2^{2}(|i-j|+2) C_{f \ell} C_{g \ell}^{3} e^{-\kappa|i-j|}$, while the remaining terms are bounded by

$$
\sum_{\substack{\ell: \vdots \\|i-\ell|>2|i-j|}} C_{f \ell} C_{g \ell}^{4} e^{-\kappa|i-\ell|} \leqq \sup _{\ell} 2^{2} C_{f \ell} C_{g \ell}^{4}\left(1-e^{-\kappa}\right)^{-1} e^{-\kappa|i-j|} .
$$

The first term in (5.9) is bounded exactly as the second, interchanging the roles of $f_{\ell}$ and $g_{\ell}$, and (5.7) follows.

Lemma 5.3. Let $\mathscr{D}^{\prime}=W\left(r_{k}^{5}, \xi_{k}-\delta-4 ;\left\{I_{k}\right\}\right)$, and let $x^{\prime}$ be as in Lemma 4.2. Then

and

$$
\sup _{\mathscr{D}^{\prime}}\left|\frac{\partial f^{\mathrm{II}}}{\partial x_{i}^{\prime}}\left(x^{\prime}\right)\right| \leqq \rho_{0}^{n} C_{1} \varepsilon_{k+1}
$$

$$
\sup \left|\frac{\partial^{2} f^{I I}}{\partial x_{i}^{\prime} \partial x_{j}^{\prime}}\left(x^{\prime}\right)\right| \leqq\left(\rho_{0}^{n} / 2^{3}\right) e^{-m\left(1-\eta_{k}\right)\left(1-6 \beta_{k}\right)|i-j|},
$$

on $W\left(r_{k}^{6}, \xi_{k}-\delta-5 ;\left\{I_{k}\right\}\right)$, where we can choose $C_{1} \sim \mathcal{O}\left(\left(\varepsilon_{k} \rho_{k}^{-1}\right)^{\alpha}\right)$, for some $\alpha>0$.

Proof. Define $\tilde{x}_{i}^{s}\left(x^{\prime}\right)$ to be either $I_{i}^{\prime}+s \Xi_{i}\left(x^{\prime}\right)$ or $\phi_{i}^{\prime}+\Delta_{i}\left(x^{\prime}\right)$ depending on the context. Since

$$
|s| \leqq 1,\left|\frac{\partial \tilde{x}_{i}^{s}}{\partial x_{j}^{\prime}}\left(x^{\prime}\right)-\delta_{i j}\right| \leqq\left|\frac{\partial \tilde{x}_{i}}{\partial x_{j}^{\prime}}\left(x^{\prime}\right)-\delta_{i j}\right|,
$$

so that Lemma 4.2 bounds derivatives of $\tilde{x}^{s}$. Let $g_{\ell}(x)=\partial f^{k[\leqq]} / \partial I_{\ell}(x),(\ell=1, \ldots, N)$. 
Then Corollary 4.5 implies $\left|g_{\ell}(x)\right| \leqq \varepsilon_{k} B^{L_{k}}$ on $W\left(r_{k}^{1}, \xi_{k}-\delta ;\left\{I_{k}\right\}\right)$. On the domain $W\left(r_{k}^{2}, \xi_{k}-\delta-1 ;\left\{I_{k}\right\}\right),(4.14),(4.15)$ and a dimensional estimate imply

$$
\left|\frac{\partial g_{\ell}}{\partial x_{i}}(x)\right| \leqq \min \left(\rho_{0}^{n} k_{0} \rho_{k+1}^{-1} \varepsilon_{k} B^{L_{k}}, \rho_{0}^{n} e^{-m\left(1-\eta_{k}\right)\left(1-2 \beta_{k}\right)|i-\ell|}\right),
$$

while (4.15) and a second dimensional estimate bounds $\left|\left(\partial^{2} g_{\ell} / \partial x_{i} \partial x_{j}\right)(x)\right|$ by

$$
\begin{aligned}
& \min \left(\rho_{0}^{n} k_{0}^{2} \rho_{k+1}^{-2} \varepsilon_{k} B^{L_{k}}, B \rho_{0}^{n} k_{0} \rho_{k+1}^{-1} e^{-m\left(1-\eta_{k}\right)\left(1-2 \beta_{k}\right)|i-\ell|},\right. \\
& \left.\quad B \rho_{0}^{n} k_{0} \rho_{k+1}^{-1} e^{-m\left(1-\eta_{k}\right)\left(1-2 \beta_{k}\right)|i-j|}\right)
\end{aligned}
$$

on $W\left(r_{k}^{3}, \xi_{k}-\delta-2 ;\left\{I_{k}\right\}\right)$. Combining these estimates with the bounds on derivatives of $x^{s}$ that come from Lemma 4.2, and applying Lemma 4.1 yields

$$
\begin{aligned}
\sup \left|\frac{\partial}{\partial x_{i}^{\prime}}\left(\frac{\partial f^{k[\leqq]}}{\partial I_{\ell}} \circ \tilde{x}^{s}\left(x^{\prime}\right)\right)\right| & \leqq B \rho_{0}^{n}(|i-\ell|+1) e^{-m\left(1-\eta_{k}\right)\left(1-4 \beta_{k}\right)|l-\ell|} \\
& \leqq B \rho_{0}^{n} e^{-m\left(1-\eta_{k}\right)\left(1-5 \beta_{k}\right)|i-\ell|}
\end{aligned}
$$

on $W\left(r_{k}^{4}, \xi_{k}-\delta-3 ;\left\{I_{k}\right\}\right)$. Note also that (4.14) and a dimensional estimate imply

and

$$
\sup _{\mathscr{D}^{\prime}}\left|\frac{\partial f^{k[\leqq]}}{\partial I_{\ell}} \circ \tilde{x}^{s}\left(x^{\prime}\right)\right| \leqq \varepsilon_{k} B^{L_{k}}
$$

$$
\sup _{\mathscr{D}^{\prime}}\left|\frac{\partial}{\partial x_{i}^{\prime}}\left(\frac{\partial f^{k[\leqq]}}{\partial I_{\ell}} \circ \tilde{x}^{s}\left(x^{\prime}\right)\right)\right| \leqq \rho_{0}^{n} k_{0} \rho_{k+1}^{-1} \varepsilon_{k} B^{L_{k}}
$$

The second half of (4.3), combined with Lemma 4.2 and the estimates above imply

$$
\sup _{\mathscr{D}^{\prime}}\left|\frac{\partial^{2}}{\partial x_{i}^{\prime} \partial x_{j}^{\prime}}\left(\frac{\partial f^{k[\leqq]}}{\partial I_{\ell}} \circ \tilde{x}^{s}\left(x^{\prime}\right)\right)\right| \leqq B \rho_{0}^{n} k_{0} \rho_{k+1}^{-1} e^{-m\left(1-\eta_{k}\right)\left(1-5 \beta_{k}\right)|i-\jmath|} .
$$

This follows by noting that the estimates above imply we can pick the constants $\kappa, C_{n}$, and $C_{n p}^{g}$ of $(4.1)$ equal to $m\left(1-\eta_{k}\right)\left(1-5 \beta_{k}\right), \rho_{0}^{n} k_{0} \rho_{k+1}^{-1} \varepsilon_{k} B^{L_{k}}$, and $2^{3} \rho_{0}^{n} k_{0} \rho_{k+1}^{-1} e^{-m\left(1-\eta_{k}\right) \beta_{k}|n-p|}$, respectively, on the domain $\mathscr{D}=W\left(r_{k}^{4}\right.$, $\xi_{k}-\delta-3 ;\left\{I_{k}\right\}$ ), while Lemma 4.2 implies we can pick the constants $C_{n p}^{1}=$ $B \rho_{0}^{n} e^{-m \beta_{k}\left(1-\eta_{k}\right)|n-p|}$, and $C_{\ell i j}^{2}=B \rho_{0}^{n} k_{0} \rho_{k+1}^{-1} e^{-m \beta_{k}\left(1-\eta_{k}\right)|i-j|}$ if $|\ell-j| \leqq 2|i-j|$ and $C_{\ell l j}^{2}=$ $B \rho_{0}^{n} k_{0} \rho_{k+1}^{-1} e^{-m\left(1-\eta_{k}\right)\left(1-4 \beta_{k}\right)|\ell-J| / 2}$ otherwise. (This last estimate follows from a dimensional estimate similar to (5.4).) Estimate (5.13) then follows by inserting these bounds in the definition of the constant $D$ in (4.3).

Note also, that (5.11) and a dimensional estimate yields

$$
\sup _{\mathscr{D}^{\prime}}\left|\frac{\partial^{2}}{\partial x_{i}^{\prime} \partial x_{j}^{\prime}}\left(\frac{\partial f^{k[\leqq]}}{\partial I_{\ell}}{ }^{\circ} \tilde{x}^{s}\left(x^{\prime}\right)\right)\right| \leqq B \rho_{0}^{n} k_{0} \rho_{k+1}^{-1} e^{-m\left(1-\eta_{k}\right)\left(1-5 \beta_{k}\right)|t-f|}
$$

The factors of $\Xi_{\ell}\left(x^{\prime}\right)$, in $f^{\mathrm{II}}$ are bounded by $\rho_{0}\left(\varepsilon_{k} \rho_{k}^{-1}\right)^{7 / 8}$ on $W\left(r_{k}^{3}, \xi_{k}-\delta-2 ;\left\{I_{k}\right\}\right)$, using (3.7) and the fact that $\Xi_{\ell}\left(I^{\prime}, \phi^{\prime}\right)=\left(\partial S / \partial \phi_{\ell}\right)\left(I^{\prime}, \phi\left(I^{\prime}, \phi^{\prime}\right)\right)$. Factors of $\partial \Xi_{\ell} / \partial x_{i}^{\prime}$ and $\partial^{2} \Xi_{\ell} / \partial x_{i}^{\prime} \partial x_{j}^{\prime}$ are bounded by Lemma 4.2 using the observation that

$$
\frac{\partial \Xi_{\ell}}{\partial x_{i}^{\prime}}=\frac{\partial \tilde{x}_{\ell}}{\partial x_{i}^{\prime}}-\delta_{\iota \ell} \quad \text { and } \quad \frac{\partial^{2} \Xi_{\ell}}{\partial x_{i}^{\prime} \partial x_{j}^{\prime}}=\frac{\partial^{2} \tilde{x}_{\ell}}{\partial x_{i}^{\prime} \partial x_{j}^{\prime}}
$$


Define $\quad \tilde{g}_{\ell}\left(x^{\prime}\right)=\partial f^{k[\leqq]} / \partial I_{\ell}^{\circ} \tilde{x}^{s}\left(x^{\prime}\right) \quad$ and $\quad f_{\ell}\left(x^{\prime}\right)=\Xi_{\ell}\left(x^{\prime}\right)$. Then inequalities (5.11)-(5.14) and the remarks in the preceding paragraph are just what we need to apply Lemma 5.2 to bound derivatives of $f_{s}^{2}\left(x^{\prime}\right) \equiv \sum_{\ell=1}^{N} \tilde{g}_{\ell}\left(x^{\prime}\right) f_{\ell}\left(x^{\prime}\right)$. Take $\kappa$ in that lemma equal to $m\left(1-\eta_{k}\right)\left(1-5 \beta_{k}\right)$, set the constants $C_{\tilde{g} \ell}=\varepsilon_{k} B^{L_{k}}, C_{\tilde{g} \ell}^{1}=$ $\rho_{0}^{n} k_{0} \rho_{k+1}^{-1} \varepsilon_{k} B^{L_{k}}, \quad C_{\tilde{g} \ell}^{2}=C_{f \ell}^{2}=B \rho_{0}^{n}, \quad C_{\tilde{g} \ell}^{3}=C_{\tilde{g} \ell}^{4}=C_{f \ell}^{3}=C_{f \ell}^{4}=B \rho_{0}^{n} k_{0} \rho_{k+1}^{-1}$, and $C_{f \ell}=$ $\rho_{0}\left(\varepsilon_{k} \rho_{k}^{-1}\right)^{7 / 8}$ and $C_{f \ell}^{1}=\rho_{0}^{n} k_{0} \rho_{k+1}^{-1} \lambda\left(\varepsilon_{0}\right)^{-2} \varepsilon_{k} B^{L_{k}}$, and let the constant $L=L_{k}$ in (5.6). We find

$$
\sup _{\mathscr{D}^{\prime}}\left|\frac{\partial f_{s}^{2}}{\partial x_{i}^{\prime}}\left(x^{\prime}\right)\right| \leqq \rho_{0}^{n} C_{1} \varepsilon_{k+1},
$$

where $C_{1}$ may be chosen $\mathcal{O}\left(\left(\varepsilon_{k} \rho_{k}^{-1}\right)^{\alpha}\right)$ for $\alpha$ some small positive constant. On $W\left(r_{k}^{6}, \xi_{k}-\delta-5 ;\left\{I_{k}\right\}\right)$,

$$
\sup \left|\frac{\partial^{2} f_{s}^{2}}{\partial x_{i}^{\prime} \partial x_{j}^{\prime}}\left(x^{\prime}\right)\right| \leqq B \rho_{0}^{n}\left[|i-j|+{ }^{\prime}\right] e^{-\kappa|i-j|} \leqq\left(\rho_{0}^{n} / 2^{3}\right) e^{-m\left(1-\eta_{k}\right)\left(1-6 \beta_{k}\right)|i-j|}
$$

In the last step in (5.16) we used the fact that we can assume $|i-j|>(1 / 8) L_{k}$, since (5.16) follows from (5.15) and a dimensional estimate otherwise. But if we now note that

$$
\left|\frac{\partial f^{\mathrm{II}}}{\partial x_{i}^{\prime}}\left(x^{\prime}\right)\right| \leqq \sup _{s}\left|\frac{\partial}{\partial x_{i}^{\prime}} f_{s}^{2}\left(x^{\prime}\right)\right| \text { and }\left|\frac{\partial^{2} f^{\mathrm{II}}}{\partial x_{i}^{\prime} \partial x_{j}^{\prime}}\left(x^{\prime}\right)\right| \leqq \sup _{s}\left|\frac{\partial^{2} f_{s}^{2}}{\partial x_{i}^{\prime} \partial x_{j}^{\prime}}\left(x^{\prime}\right)\right|
$$

we see that (5.10) follows from (5.15) and (5.16).

Finally we address derivatives of $f^{1}$.

Lemma 5.4. Let $\tilde{x}^{s}\left(x^{\prime}\right)$ be as defined in the proof of Lemma 5.3. Define

$$
h_{n}\left(x^{\prime}\right)=\sum_{l=1}^{N} \frac{\partial^{2} h^{k}}{\partial I_{\ell} \partial I_{n}} \circ \tilde{x}^{s}\left(x^{\prime}\right) \cdot \Xi_{\ell}\left(x^{\prime}\right)
$$

Then on $W\left(r_{k}^{6}, \xi_{k}-\delta-5 ;\left\{I_{k}\right\}\right)$,

$$
\begin{aligned}
\sup \left|h_{n}\left(x^{\prime}\right)\right| & \leqq B \rho_{0}\left(\varepsilon_{k} \rho_{k}^{-1}\right)^{7 / 8}, \\
\sup \left|\frac{\partial h_{n}}{\partial x_{i}^{\prime}}\left(x^{\prime}\right)\right| & \leqq \min \left\{\begin{array}{l}
B \rho_{0}^{n} k_{0} \rho_{k+1}^{-1}\left(\varepsilon_{k} \rho_{k}^{-1}\right)^{7 / 8} \\
\rho_{0}^{n} e^{-m\left(1-\eta_{k}\right)\left(1-7 \beta_{k}\right)|i-n|}
\end{array}\right.
\end{aligned}
$$

and

$$
\sup \left|\frac{\partial^{2} h_{n}}{\partial x_{i}^{\prime} \partial x_{j}^{\prime}}\left(x^{\prime}\right)\right| \leqq \min \left\{\begin{array}{l}
B \rho_{0}^{n} k_{0} \rho_{k+1}^{-1} e^{-m\left(1-\eta_{k}\right)\left(1-7 \beta_{k}\right)|i-n|} \\
B \rho_{0}^{n} k_{0}^{2} \rho_{k+1}^{-2} e^{-m\left(1-\eta_{k}\right)\left(1-7 \beta_{k}\right)|i-j|}
\end{array}\right.
$$

Assuming Lemma 5.4 holds for the moment we prove

Lemma 5.5. Let $\mathscr{D}=W\left(r_{k}^{7}, \xi_{k}-\delta-6 ;\left\{I_{k}\right\}\right)$. Then

$$
\sup _{\mathscr{D}}\left|\frac{\partial f^{1}}{\partial x_{i}^{\prime}}\left(x^{\prime}\right)\right| \leqq \rho_{0}^{n} C_{1} \varepsilon_{k+1}
$$


and

$$
\sup _{\mathscr{c}}\left|\frac{\partial^{2} f^{\mathrm{I}}}{\partial x_{i}^{\prime} \partial x_{j}^{\prime}}\left(x^{\prime}\right)\right| \leqq\left(\rho_{0}^{n} / 2^{3}\right) e^{-m\left(1-\eta_{k}\right)\left(1-8 \beta_{k}\right)|i-j|}
$$

where we can choose $C_{1} \sim \mathcal{O}\left(\left(\varepsilon_{k} \rho_{k}^{-1}\right)^{\alpha}\right)$ for $\alpha$ some small positive constant.

Proof. Note that

$$
\left|\frac{\partial}{\partial x_{i}^{\prime}} f^{\mathrm{I}}\left(x^{\prime}\right)\right| \leqq \sup _{s}\left|\frac{\partial}{\partial x_{i}^{\prime}}\left(\sum_{n=1}^{N} h_{n}\left(x^{\prime}\right) \Xi_{n}\left(x^{\prime}\right)\right)\right|
$$

and

$$
\left|\frac{\partial^{2}}{\partial x_{i}^{\prime} \partial x_{j}^{\prime}} f^{\mathrm{I}}\left(x^{\prime}\right)\right| \leqq \sup _{s}\left|\frac{\partial^{2}}{\partial x_{i}^{\prime} \partial x_{j}^{\prime}}\left(\sum_{n=1}^{N} h_{n}\left(x^{\prime}\right) \Xi_{n}\left(x^{\prime}\right)\right)\right| .
$$

We apply Lemma 5.2, with $f_{\ell}=h_{\ell}$ (the function defined in Lemma 5.4), and $g_{\ell}=\Xi_{\ell}$, to derive bounds on the right hand side of these inequalities. (Our bounds are independent of s.) Bounds on $f_{\ell}$ are given in Lemma 5.4, while bounds on $\Xi_{\ell}$ and its derivatives were discussed in the proof of Lemma 5.3. Pick the domain $\mathscr{D}$ in Lemma 5.2 to be $W\left(r_{k}^{6}, \xi_{k}-\delta-5 ;\left\{I_{k}\right\}\right)$, and choose $\kappa=m\left(1-\eta_{k}\right)\left(1-7 \beta_{k}\right), C_{g \ell}=C_{f \ell}=$ $B \rho_{0}^{n}\left(\varepsilon_{k} \rho_{k}^{-1}\right)^{7 / 8}, C_{g \ell}^{1}=C_{f f}^{1}=B \rho_{0}^{n} k_{0} \rho_{k+1}^{-1}\left(\varepsilon_{k} \rho_{k}^{-1}\right)^{7 / 8}, C_{f l}^{2}=C_{g \ell}^{2}=B \rho_{0}^{n}, C_{f \ell}^{3}=C_{f \ell}^{4}=C_{g \ell}^{3}=$ $C_{g \ell}^{4}=B \rho_{0}^{n} \kappa_{0}^{2} \rho_{k+1}^{-2}$, and the constant $L$ in (5.6) to be $L_{k}$. Then (5.20) follows easily from (5.6) and (5.7).

Combine Proposition 5.1 with Lemmas 5.3 and 5.5., and take the definition of $f^{k+1}\left(I^{\prime}, \phi^{\prime}\right)$ in (3.17) to obtain

Corollary 5.6. Let $\mathscr{D}=W\left(r_{k}^{7}, \xi_{k}-\delta-6 ;\left\{I_{k}\right\}\right) \supset W\left(\rho^{k+1}, \xi_{k+1} ;\left\{I_{k+1}\right\}\right)$. Then

$$
\sup _{\mathscr{D}}\left|\frac{\partial^{2} f}{\partial x_{i}^{\prime} \partial x_{j}^{\prime}}\left(x^{\prime}\right)\right| \leqq \rho_{0}^{n} e^{-m\left(1-\eta_{k+1}\right)|t-\jmath|} \text {. }
$$

This implies that (2.10) holds for the Hamiltonian $H^{k+1}$. All that remains is to verify that (2.5) and (2.6) also hold for $f^{k+1}$, which we do in the next section.

We now prove Lemma 5.4. By (2.8) and (2.9),

$$
\left|\frac{\partial^{2} h^{k}}{\partial I_{\ell} \partial I_{n}} \circ \tilde{x}^{s}\left(x^{\prime}\right)-\delta_{\ell n}\right| \leqq e^{-m\left(1-\eta_{k}\right)|\ell-n|}
$$

on $W\left(r_{k}^{3}, \xi_{k}-\delta-2 ;\left\{I_{k}\right\}\right)$. Since $\left|\Xi_{\ell}\left(x^{\prime}\right)\right| \leqq \rho_{0}\left(\varepsilon_{k} \rho_{k}^{-1}\right)^{7 / 8}$, on this same domain, we see that $\left|h_{n}\left(x^{\prime}\right)\right| \leqq B \rho_{0}\left(\varepsilon_{k} \rho_{k}^{-1}\right)^{7 / 8}$ as claimed in (5.17). A dimensional estimate immediately yields the first estimate in (5.18) on the smaller domain $W\left(r_{k}^{4}, \xi_{k}-\delta-3 ;\left\{I_{k}\right\}\right)$.

Note that (2.8) and (2.9) coupled with a pair of dimensional estimates imply

$$
\sup \left|\frac{\partial}{\partial x_{i}}\left(\frac{\partial^{2} h^{k}}{\partial I_{\ell} \partial I_{n}}(x)\right)\right| \leqq \min \left\{\begin{array}{l}
B \rho_{0}^{n} k_{0} \rho_{k+1}^{-1} e^{-m\left(1-\eta_{k}\right)|t-n|} \\
B \rho_{0}^{n} k_{0} \rho_{k+1}^{-1} e^{-m\left(1-\eta_{k}\right)|\ell-n|}
\end{array}\right.
$$

and

$$
\sup \left|\frac{\partial^{2}}{\partial x_{i} \partial x_{j}}\left(\frac{\partial^{2} h^{k}}{\partial I_{\ell} \partial I_{n}}(x)\right)\right| \leqq \min \left\{\begin{array}{l}
B \rho_{0} k_{0}^{2} \rho_{k+1}^{-2} e^{-m\left(1-\eta_{k}\right)\left|I_{-}-\ell\right|} \\
B \rho_{0} k_{0}^{2} \rho_{k+1}^{-2} e^{-m\left(1-\eta_{k}\right)|1-\jmath|}
\end{array}\right.
$$


where the suprema run over $W\left(r_{k}^{1}, \xi_{k}-\delta\right.$; $\left.\left\{I_{k}\right\}\right)$ and $x$ is as in (1.14). Now apply Lemma 4.1, with the domains $\mathscr{D}=W\left(r_{k}^{3}, \xi_{k}-\delta-2 ;\left\{I_{k}\right\}\right)$ and $\mathscr{D}^{\prime}=W\left(r_{k}^{4}, \xi_{k}-\delta-3\right.$; $\left.\left\{I_{k}\right\}\right)$ and the function $g=\partial^{2} h^{k} / \partial I_{\ell} \partial I_{n}$. The bounds on $g$ and its derivatives coming from (5.23)-(5.25) and the bounds on $\tilde{x}^{s}$ from Lemma 4.2 combined with the second estimate of (4.3) imply

$$
\sup _{\mathscr{D}^{\prime}}\left|\frac{\partial^{2}}{\partial x_{i}^{\prime} \partial x_{j}^{\prime}}\left(\frac{\partial^{2} h^{k}}{\partial I_{\ell} \partial I_{n}} \circ \tilde{x}^{s}\left(x^{\prime}\right)\right)\right| \leqq B \rho_{0}^{n} k_{0}^{2} \rho_{k+1}^{-2} e^{-m\left(1-\eta_{k}\right)\left(1-5 \beta_{k}\right)|i-j|},
$$

while the first estimate in (4.3) implies

$$
\sup _{\mathscr{D}^{\prime}}\left|\frac{\partial}{\partial x_{i}^{\prime}}\left(\frac{\partial^{2} h^{k}}{\partial I_{\ell} \partial I_{n}} \circ \tilde{x}^{s}\left(x^{\prime}\right)\right)\right| \leqq B \rho_{0}^{n} k_{0} \rho_{k+1}^{-1} e^{-m\left(1-\eta_{k}\right)\left(1-5 \beta_{k}\right)|i-\ell|} .
$$

Because of the symmetry between $\ell$ and $n$, (5.27) also holds with $\ell$ and $n$ interchanged. Furthermore, (5.23) and (5.27) plus a pair of dimensional estimates on $W\left(r_{k}^{5}, \xi_{k}-\delta-4 ;\left\{I_{k}\right\}\right)$ imply

$$
\left|\frac{\partial^{2}}{\partial x_{i} \partial x_{j}}\left(\frac{\partial^{2} h^{k}}{\partial I_{\ell} \partial I_{n}} \circ \tilde{x}^{s}\left(x^{\prime}\right)\right)\right| \leqq \min \left\{\begin{array}{l}
B \rho_{0}^{n} k_{0}^{2} \rho_{k+1}^{-2} e^{-m\left(1-\eta_{k}\right)\left(1-5 \beta_{k}\right)|i-\ell|} \\
B \rho_{0}^{n} k_{0}^{2} \rho_{k+1}^{-2} e^{-m\left(1-\eta_{k}\right)\left(1-5 \beta_{k}\right)|i-n|},
\end{array}\right.
$$

and

$$
\left|\frac{\partial}{\partial x_{i}}\left(\frac{\partial^{2} h^{k}}{\partial I_{\ell} \partial I_{n}} \circ \tilde{x}^{s}\left(x^{\prime}\right)\right)\right| \leqq B \rho_{0} k_{0} \rho_{k+1}^{-1} e^{-m\left(1-\eta_{k}\right)|\ell-n|} .
$$

Now apply Lemma 5.2, with $f_{\ell}\left(x^{\prime}\right)=\left(\partial^{2} h^{k} / \partial I_{\ell} \partial I_{n}\right) \circ \tilde{x}^{s}\left(x^{\prime}\right), g_{\ell}\left(x^{\prime}\right)=\Xi_{\ell}\left(x^{\prime}\right)$ and $\mathscr{D}=W\left(r_{k}^{5}, \xi_{k}-\delta-4 ;\left\{I_{k}\right\}\right)$. Then (5.23) and (5.26)-(5.28) bound $f_{\ell}$ and its derivatives while (3.7) and Lemma 4.2, coupled with the observations in the proof of Lemma 5.3 bound $g_{\ell}$ and its derivatives. Estimate (5.6) implies

$$
\sup _{\mathscr{D}}\left|\frac{\partial}{\partial x_{i}^{\prime}} h_{n}\left(x^{\prime}\right)\right| \leqq B \rho_{0}^{n} e^{-m\left(1-\eta_{k}\right)\left(1-6 \beta_{k}\right)|i-n|},
$$

which implies the second estimate in (5.18). The first of the bounds in (5.19) follows from (5.29) by a dimensional estimate. Applying Lemma 5.2 a second time we find (5.7) implies

$$
\sup _{\mathscr{D}}\left|\frac{\partial^{2}}{\partial x_{i}^{\prime} \partial x_{j}^{\prime}} h_{n}\left(x^{\prime}\right)\right| \leqq B \rho_{0}^{n} k_{0}^{2} \rho_{k+1}^{-2} e^{-m\left(1-\eta_{k}\right)\left(1-7 \beta_{k}\right)|i-j|},
$$

which completes the proof of Lemma 5.4. We note that in applying Lemma 5.2 to derive (5.29) and (5.30) it is necessary to make different choices of the constant $\kappa$, and hence of the other constants, in the two cases but the details are not difficult to work out so we omit them.

\section{The Strength of the "Renormalized" Interactions}

In the present section we verify that (2.5) and (2.6) hold for the Hamiltonian $H^{k+1}$. First note that the fundamental theorem of calculus allows us to write 


$$
\begin{aligned}
f^{k, \mathrm{r}}\left(I^{\prime}\right. & \left.+\Xi\left(I^{\prime}, \phi^{\prime}\right), \phi^{\prime}+\Delta\left(I^{\prime}, \phi^{\prime}\right)\right) \\
= & f^{k, \mathrm{r}}\left(I^{\prime}, \phi^{\prime}\right)+\sum_{\ell=1}^{N} \int_{0}^{1} d s \frac{\partial f^{k, \mathrm{r}}}{\partial I_{\ell}}\left(I^{\prime}+s \Xi, \phi^{\prime}+s \Delta\right) \cdot \Xi_{\ell}\left(I^{\prime}, \phi^{\prime}\right) \\
& +\sum_{\ell=1}^{N} \int_{0}^{1} d s \frac{\partial f^{k, \mathrm{r}}}{\partial \phi_{\ell}}\left(I^{\prime}+s \Xi, \phi^{\prime}+s \Delta\right) \cdot \Delta_{\ell}\left(I^{\prime}, \phi^{\prime}\right) \\
\equiv & f^{k, \mathrm{r}}\left(I^{\prime}, \phi^{\prime}\right)+\delta f^{k, \mathrm{r}}\left(I^{\prime}, \phi^{\prime}\right)
\end{aligned}
$$

and

$$
\begin{aligned}
f^{\mathrm{IV}}\left(I^{\prime}, \phi^{\prime}\right)= & f^{4}\left(I^{\prime}, \phi^{\prime}\right)+\sum_{\ell=1}^{N} \int_{0}^{1} d s \frac{\partial f^{4}}{\partial I_{\ell}}\left(I^{\prime}+s \Xi, \phi^{\prime}+s \Delta\right) \cdot \Xi_{\ell}\left(I^{\prime}, \phi^{\prime}\right) \\
& +\sum_{\ell=1}^{N} \int_{0}^{1} d s \frac{\partial f^{4}}{\partial \phi_{\ell}}\left(I^{\prime}+s \Xi, \phi^{\prime}+s \Delta\right) \cdot \Delta_{\ell}\left(I^{\prime}, \phi^{\prime}\right) \\
\equiv & f^{4}\left(I^{\prime}, \phi^{\prime}\right)+\delta f^{4}\left(I^{\prime}, \phi^{\prime}\right) .
\end{aligned}
$$

Proposition 6.1. On $W\left(r_{k}^{6}, \xi_{k}-\delta-5 ;\left\{I_{k}\right\}\right)$

and

$$
\sup \left|\frac{\partial}{\partial x_{i}^{\prime}}\left(\delta f^{4}\left(x^{\prime}\right)\right)\right| \leqq C_{1} \rho_{0}^{n} \varepsilon_{k+1}
$$

$$
\sup \left|\frac{\partial}{\partial x_{i}^{\prime}}\left(\delta f^{k, r}\left(x^{\prime}\right)\right)\right| \leqq\left\{\begin{array}{l}
\rho_{0}^{n} k_{0} \varepsilon_{k} \rho_{k+1}^{-1} B^{L_{k}} \quad \text { if } \quad i \in S^{m}, m=0, \ldots, k \\
\rho_{0}^{n} C_{1} \varepsilon_{k+1} \quad \text { if } i \notin S^{m}, m=0, \ldots, k,
\end{array}\right.
$$

where $C_{1} \sim \mathcal{O}\left(\left(\varepsilon_{k} \rho_{k}^{-1}\right)^{\alpha}\right)$, for $\alpha$ some small positive constant.

Now note that

$$
\begin{aligned}
f_{v}^{k+1}\left(I^{\prime}\right)= & \int d \phi^{\prime} f^{k+1}\left(I^{\prime}, \phi^{\prime}\right) e^{i v \cdot \phi^{\prime}} \\
= & \int d \phi^{\prime}\left\{f^{k, \mathrm{r}}\left(I^{\prime}, \phi^{\prime}\right)+f^{4}\left(I^{\prime}, \phi^{\prime}\right)+\delta f^{k, \mathrm{r}}\left(I^{\prime}, \phi^{\prime}\right)\right. \\
& \left.+\delta f^{4}\left(I^{\prime}, \phi^{\prime}\right)+f^{\mathrm{I}}\left(I^{\prime}, \phi^{\prime}\right)+f^{\mathrm{II}}\left(I^{\prime}, \phi^{\prime}\right)+f^{\mathrm{III}}\left(I^{\prime}, \phi^{\prime}\right)\right\} e^{i v \cdot \phi^{\prime}} .
\end{aligned}
$$

(Here, $\int d \phi^{\prime} \equiv \prod_{i=1}^{N}\left(\frac{1}{2 \pi}\right) \int_{-\pi}^{\pi} d \phi_{i}^{\prime}$.) But

$$
\int d \phi^{\prime}\left\{f^{k, \mathrm{r}}\left(I^{\prime}, \phi^{\prime}\right)+f^{4}\left(I^{\prime}, \phi\right)\right\} e^{i v \cdot \phi^{\prime}}=f_{v}^{k}\left(I^{\prime}\right)
$$

if $v \in\left[\bigcup_{m=0}^{k-1}\left(R_{p}^{m} \cup R_{s}^{m}\right)\right] \cup R_{p}^{k}$, and zero otherwise. Note that if $v \notin \bigcup_{m=0}^{k}\left[R_{p}^{m} \cup R_{s}^{m}\right]$, then either there exists $\ell \in \operatorname{supp} v$ such that $\ell \notin S^{m}, m=0, \ldots, k$, or $v \notin \mathbb{X}_{k+1}$. In the first of these cases, notice that Proposition 6.1, Proposition 5.1, and Lemmas 5.4 and 5.5. when combined with Cauchy's theorem, and the observation that for such a $v$, $\int d \phi^{\prime}\left\{f^{k, \mathrm{r}}\left(I^{\prime}, \phi^{\prime}\right)+f^{4}\left(I^{\prime}, \phi^{\prime}\right)\right\} e^{i v \cdot \phi^{\prime}}=0$, imply that (on $W\left(r_{k}^{7}, \xi_{k}-\delta-6 ;\left\{I_{k}\right\}\right)$ )

$$
\left|\frac{\partial}{\partial I_{j}^{\prime}} f_{v}^{k+1}\left(I^{\prime}\right)\right|+\rho_{0}^{-1}\left|v_{j} f_{v}^{k+1}\left(I^{\prime}\right)\right| \leqq C_{1} \rho_{0} k_{0} \varepsilon_{k+1} \rho_{k+1}^{-1} e^{-\left(\xi_{k}-\delta-6\right)|v|} .
$$

In the second case, Corollary 5.6. combined with the estimates above, implies

$$
\begin{aligned}
& \left|\frac{\partial}{\partial I_{j}^{\prime}} f_{v}^{k+1}\left(I^{\prime}\right)\right|+\rho_{0}^{-1}\left|v_{j} f_{v}^{k+1}\left(I^{\prime}\right)\right| \\
& \quad \leqq \min \left(B \rho_{0}^{2} k_{0} \rho_{k+1}^{-1} e^{-m\left(1-\eta_{k+1}\right) d(\text { supp v) }} e^{-\left(\xi_{k}-\delta-6\right)|v|}, \rho_{0} k_{0} \varepsilon_{k} \rho_{k+1}^{-1} B^{L_{k}} e^{-\left(\xi_{k}-\delta-6\right)|v|}\right),
\end{aligned}
$$


on the same domain, from which it follows that if $\nu \notin \bigcup_{m=0}^{k}\left[R_{p}^{m} \cup R_{s}^{m}\right]$,

$$
\left|\frac{\partial}{\partial I_{j}^{\prime}} f_{v}^{k+1}\left(I^{\prime}\right)\right|+\rho_{0}^{-1}\left|v_{j} f_{v}^{k+1}\left(I^{\prime}\right)\right| \leqq C_{2} \varepsilon_{k+1} e^{-\left(\xi_{k}-2 \delta-6\right)|v|},
$$

with both $C_{1}$ and $C_{2} \sim \mathcal{O}\left(\left(\varepsilon_{k} \rho_{k}^{-1}\right)^{\alpha}\right)$.

Remark. Inequality (6.7) does not imply (6.8) in the case $v=0$-we treat this special case at the end of this section.

Next remark that on $W\left(\rho^{k+1}, \xi_{k+1} ;\left\{I_{k+1}\right\}\right)$,

$$
\left|\frac{\partial f^{k+1, \mathrm{nr}}}{\partial I_{j}^{\prime}}\right|+\rho_{0}^{-1}\left|\frac{\partial f^{k+1, \mathrm{nr}}}{\partial \phi_{j}^{\prime}}\right| \leqq \sum_{v \notin R^{\prime}}\left(\left|\frac{\partial f_{v}^{k+1}}{\partial I_{j}^{\prime}}\right|+\rho_{0}^{-1}\left|v_{j} f_{v}^{k+1}\right|\right) e^{\xi_{k+1}|v|}
$$

with $\tilde{R}^{\prime}=\bigcup_{m=0}^{k}\left(R_{p}^{m} \cup R_{s}^{m}\right)$. Corollary 5.6, (6.8), and Cauchy's theorem imply the summand in (6.9) is bounded by $\min \left(C_{2} \varepsilon_{k+1} e^{-\delta|v|}, \rho_{0} e^{-m\left(1-\eta_{k+1}\right)|j-\ell|} e^{-\delta|v|}\right)$, where $\ell$ is the point is supp $v$ farthest from $j$. If we now apply Proposition 4.3 we see that on $W\left(\rho^{k+1}, \xi_{k+1} ;\left\{I_{k+1}\right\}\right)$,

$$
\sup \left\{\left|\frac{\partial f^{k+1, \mathrm{nr}}}{\partial I_{j}^{\prime}}\right|+\rho_{0}^{-1}\left|\frac{\partial f^{k+1, \mathrm{nr}}}{\partial \phi_{j}}\right|\right\} \leqq \varepsilon_{k+1},
$$

so (2.5) applies to the Hamiltonian $H^{k+1}$.

Next assume that $j \in S^{m}$ for some $m=0, \ldots, k$. Then on $W\left(\rho^{k+1}, \xi_{k+1},\left\{I_{k+1}\right\}\right)$,

$$
\begin{aligned}
& \left|\frac{\partial f^{k+1, \mathrm{r}}}{\partial I_{j}^{\prime}}\right|+\rho_{0}^{-1}\left|\frac{\partial f^{k+1, \mathrm{r}}}{\partial \phi_{j}^{\prime}}\right| \leqq\left|\frac{\partial f^{k, \mathrm{r}}}{\partial I_{j}^{\prime}}\right|+\rho_{0}^{-1}\left|\frac{\partial f^{k, \mathrm{r}}}{\partial \phi_{j}^{\prime}}\right| \\
& \quad+\sum_{v \in \tilde{R}}\left\{\left|\frac{\partial}{\partial I_{j}^{\prime}}\left(f_{v}^{k+1}\left(I^{\prime}\right)-f_{v}^{k}\left(I^{\prime}\right)\right)\right|+\rho_{0}^{-1}\left|v_{j}\left(f_{v}^{k+1}\left(I^{\prime}\right)-f_{v}^{k}\left(I^{\prime}\right)\right)\right|\right\} e^{\xi_{k+1}|v|} .
\end{aligned}
$$

By Proposition 6.1, Corollary 5.6, (2.10), (6.5) and Cauchy's theorem, the summand on the right hand side of (6.11) can be bounded by

$$
\min \left(\rho_{0}^{n} k_{0} \varepsilon_{k} \rho_{k+1}^{-1} B^{L_{k}} e^{-\delta|v|}, 2 \rho_{0} e^{-m\left(1-\eta_{k+1}\right)|j-\ell|} e^{-\delta|v|}\right),
$$

where $\ell$ is the point in supp $v$ farthest from $j$. Apply Proposition 4.3 and bound this sum by $\rho_{0} k_{0} \varepsilon_{k} \rho_{k+1}^{-1} B^{L_{k+1}}$. By (2.6)

$$
\left|\frac{\partial f^{k, \mathrm{r}}}{\partial I_{j}^{\prime}}\right|+\rho_{0}^{-1}\left|\frac{\partial f^{k, \mathrm{r}}}{\partial \phi_{j}^{\prime}}\right| \leqq A(k, j)
$$

and since $j \in S^{m}, A(k, j)+\rho_{0} k_{0} \varepsilon_{k} \rho_{k+1}^{-1} B^{L_{k+1}} \leqq A(k+1, j)$, so (2.6) holds for the Hamiltonian $H^{k+1}$ if $j \in \bigcup_{m=0}^{k} S^{m}$ and it remains only to check that it holds for $j \notin \bigcup_{m=0}^{k} S^{m}$.

If $j \notin \bigcup_{m=0}^{k} S^{m}$, and $v \in \bigcup_{m=0}^{k-1}\left(R_{p}^{m} \cup R_{s}^{m}\right)$, the definition of $R_{s}^{k-1}$ implies there must be some $\ell \in \operatorname{supp} v$ such that $\operatorname{dist}(j, \ell)>L_{k}$. Thus applying (2.10) and Cauchy's theorem we see that if $v \in \bigcup_{m=0}^{k-1}\left[R_{p}^{m} \cup R_{s}^{m}\right]$, 


$$
\begin{aligned}
& \left\{\left|\frac{\partial f_{v}^{k}}{\partial I_{j}^{\prime}}\left(I^{\prime}\right)\right|+\rho_{0}^{-1}\left|v_{j} f_{v}^{k}\left(I^{\prime}\right)\right|\right\} \leqq 2 \rho_{0} e^{-m\left(1-\eta_{k}\right)|j-\ell|} e^{-\xi_{k}|v|} \\
& \leqq \min \left(B \rho_{0}\left(\varepsilon_{0} \rho_{0}^{-1}\right)^{(8 / 5)(3 / 2)^{k}} e^{-\xi_{k}|v|}, B \rho_{0} e^{-m\left(1-\eta_{k}|| j-\ell \mid\right.} e^{-\xi_{k}|v|}\right)
\end{aligned}
$$

on $W\left(\rho^{k}, \xi_{k} ;\left\{I_{k}\right\}\right)$. Here $\ell$ is the point in $\operatorname{supp} v$ farthest from $j$, and the second inequality used our observation that $|j-\ell|>L_{k}$. Hence, applying Proposition 4.3 we find

$$
\begin{aligned}
\sup \left\{\left|\frac{\partial f^{k, \mathrm{r}}}{\partial I_{j}^{\prime}}\right|+\rho_{0}^{-1}\left|\frac{\partial f^{k, \mathrm{r}}}{\partial \phi_{j}^{\prime}}\right|\right\} & \leqq \sum_{v \in R^{\prime}}\left\{\left|\frac{\partial f_{v}^{k}}{\partial I_{j}^{\prime}}\right|+\rho_{0}^{-1}\left|v_{j} f_{v}^{k}\right|\right\} e^{\xi_{k+1}|v|} \\
& \leqq \rho_{0}\left(\varepsilon_{0} \rho_{0}^{-1}\right)^{(8 / 5)(3 / 2)^{k}} B^{L_{k+1}}
\end{aligned}
$$

on $W\left(\rho^{k+1}, \xi_{k+1} ;\left\{I_{k+1}\right\}\right)$. Combining this with (6.11) we see that if $j \notin \bigcup_{m=0}^{k} S^{m}$,

$$
\begin{aligned}
& \left\{\left|\frac{\partial f^{k+1, \mathrm{r}}}{\partial I_{j}^{\prime}}\right|+\rho_{0}^{-1}\left|\frac{\partial f^{k+1, \mathrm{r}}}{\partial \phi_{j}^{\prime}}\right|\right\} \\
& \leqq \rho_{0}\left(\varepsilon_{0} \rho_{0}^{-1}\right)^{(8 / 5)(3 / 2)^{k}} B^{L_{k+1}}+C_{1} \varepsilon_{k+1} B^{L_{k+1}}, \leqq \varepsilon_{k+1},
\end{aligned}
$$

so that (2.6) holds for all sites $j$, and the Hamiltonian satisfies the estimates (2.5)(2.10). Note that in (6.14) we used the fact that on $W\left(r_{k}^{7}, \xi_{k}-\delta-6 ;\left\{I_{k}\right\}\right)$,

$$
\begin{aligned}
\left|\frac{\partial}{\partial I_{j}^{\prime}}\left(f_{v}^{k+1}\left(I^{\prime}\right)-f_{v}^{k}\left(I^{\prime}\right)\right)\right|= & \mid \int d \phi^{\prime} \frac{\partial}{\partial I_{j}^{\prime}}\left(\delta f^{k, \mathrm{r}}\left(I^{\prime}, \phi^{\prime}\right)+\delta f^{4}\left(I^{\prime}, \phi^{\prime}\right)\right. \\
& \left.+f^{\mathrm{I}}\left(I^{\prime}, \phi^{\prime}\right)+f^{\mathrm{II}}\left(I^{\prime}, \phi^{\prime}\right)+f^{\mathrm{III}}\left(I^{\prime}, \phi^{\prime}\right)\right) e^{i v \cdot \phi^{\prime}} \mid \\
\leqq & C_{1} \varepsilon_{k+1} e^{-\left(\xi_{k}-\delta-5\right)|v|}
\end{aligned}
$$

if $j \notin S^{m}, m=0, \ldots, k$, and analogously for $\rho_{0}^{-1}\left|v_{j}\left(f_{v}^{k+1}\left(I^{\prime}\right)-f_{v}^{k}\left(I^{\prime}\right)\right)\right|$, and then applied Proposition 4.3 to bound the sum in (6.11) by $C_{1} \varepsilon_{k+1} B^{L_{k+1}}$.

We complete this section with the proof of Proposition 6.1. The first inequality in (6.3) is an easy application of Lemma 5.2. Let $x$ be as in (1.14), $x^{\prime}$ as in Lemma 4.2, and redefine $\tilde{x}_{i}^{s}\left(x^{\prime}\right)$ as either $I_{i}^{\prime}+s \Xi_{i}\left(x^{\prime}\right)$ or $\varphi_{i}^{\prime}+s \Delta_{i}\left(x^{\prime}\right)$ depending on context. Let the functions $f_{\ell}\left(x^{\prime}\right)$ and $g_{\ell}\left(x^{\prime}\right)$ of Lemma 5.2 be defined by $f_{\ell}\left(x^{\prime}\right)=\left(\partial f^{4} / \partial x_{\ell}\right) \circ \tilde{x}^{s}\left(x^{\prime}\right), g_{\ell}\left(x^{\prime}\right)=$ $\Xi_{\ell}\left(x^{\prime}\right)$ in the second term on the right hand side of $(6.2)$, and $g_{\ell}\left(x^{\prime}\right)=\Delta_{\ell}\left(x^{\prime}\right)$ in the third term on the right hand side of (6.2). Let $\mathscr{D}$, the domain in Lemma 5.2 be $W\left(r_{k}^{5}, \xi_{k}-\delta-4 ;\left\{I_{k}\right\}\right)$. We will give estimates on $\left|\left(\partial / \partial x_{i}^{\prime}\right)\left(\sum_{\ell} f_{\ell}\left(x^{\prime}\right) g_{\ell}\left(x^{\prime}\right)\right)\right|$ which are independent of $s \in[0,1]$, and which therefore bound $\left|\left(\partial / \partial x_{i}^{\prime}\right)\left(\delta f^{4}\left(x^{\prime}\right)\right)\right|$. By Corollary 4.5 and a dimensional estimate we choose the constants $C_{f \ell}=\rho_{0}^{n} \varepsilon_{k} B^{L_{k}}$ and $C_{f \ell}^{1}=$ $\rho_{0}^{n} \varepsilon_{k} k_{0} \rho_{k+1}^{-1} B^{L_{k}}$. Applying Lemma 4.1, with the bounds on derivatives of the map $\tilde{x}^{s}$ that come from Lemma $4.2\left(\right.$ since $\left.\left(\partial \tilde{x}_{\ell}^{s} / \partial x_{j}^{\prime}\right)-\tilde{\delta}_{\ell j}=s\left(\left(\partial \tilde{x}_{\ell} / \partial x_{j}^{\prime}\right)-\tilde{\delta}_{\ell}\right)\right)$ we see that

$$
\left|\frac{\partial f_{\ell}}{\partial x_{i}^{\prime}}\left(x^{\prime}\right)\right|=\left|\frac{\partial}{\partial x_{i}^{\prime}}\left(\frac{\partial f^{4}}{\partial x_{\ell}} \circ \tilde{x}^{s}\left(x^{\prime}\right)\right)\right| \leqq B \rho_{0}^{n} e^{-m\left(1-\eta_{k}\right)\left(1-5 \beta_{k}\right)|i-\ell|},
$$

on $W\left(r_{k}^{4}, \xi_{k}-\delta-3 ;\left\{I_{k}\right\}\right)$, so we can take $C_{f \ell}^{2}=B \rho_{0}^{n}$ and $\kappa=m\left(1-\eta_{k}\right)\left(1-5 \beta_{k}\right)$. Since $\Xi_{\lambda}\left(x^{\prime}\right)=\left(\partial S / \partial \phi_{\ell}\right)\left(I^{\prime}, \phi\left(I^{\prime}, \phi^{\prime}\right)\right)$ and $\Delta_{\ell}\left(x^{\prime}\right)=\left(\partial S / \partial I_{\ell}^{\prime}\right)\left(I^{\prime}, \phi\left(I^{\prime}, \phi^{\prime}\right)\right),(3.7)$ and a dimensional estimate imply we can pick $C_{g \ell}=\rho_{0}^{n}\left(\lambda\left(\varepsilon_{0}\right)\right)^{-2} \varepsilon_{k} B^{L_{k}}, C_{g \ell}^{1}=\rho_{0}^{n}\left(\lambda\left(\varepsilon_{0}\right)\right)^{-2} \times$ $k_{0} \varepsilon_{k} \rho_{k+1}^{-1} B^{L_{k}}$, while Lemma 4.2 implies that $C_{g \ell}^{2}=\rho_{0}^{n}$ with the same choice of $\kappa$ as 
above, (since $\partial g_{\ell} / \partial x_{i}^{\prime}=\left(\partial \tilde{x}_{\ell} / \partial x_{i}^{\prime}\right)-\delta_{\ell i}$, with $\tilde{x}$ as in Lemma 4.2). Combining these observations with (5.6) and setting $L=L_{k}$ in that expression we obtain the first inequality in (6.3).

To prove the second estimate in (6.3), let $f_{\ell}\left(x^{\prime}\right)=\left(\partial f^{k, \mathrm{r}} / \partial x_{\ell}\right) \circ \tilde{x}^{s}\left(x^{\prime}\right), g_{\ell}\left(x^{\prime}\right)$ be just as above, and set $\mathscr{D}=W\left(r_{k}^{6}, \xi_{k}-\delta-5 ;\left\{I_{k}\right\}\right)$. Once again we will prove estimates on $\left|\left(\partial / \partial x_{i}^{\prime}\right)\left(\sum_{\ell} f_{\ell}\left(x^{\prime}\right) g_{\ell}\left(x^{\prime}\right)\right)\right|$ which are independent of $s \in[0,1]$, and thus imply the second inequality in (6.3). Combining Lemma 4.2 with Corollary 4.5 and Lemma 4.1 we see that we can set $C_{f \ell}^{2}=C_{g \ell}^{2}=B \rho_{0}^{n}$, and $\kappa=m\left(1-\eta_{k}\right)\left(1-6 \beta_{k}\right)$, in Lemma 5.2.

We now consider two cases.

Case 1. $\ell \notin \bigcup_{m=0}^{k-1} S^{m}$. We can set $C_{f \ell}=\rho_{0}\left(\varepsilon_{k} \rho_{k}^{-1}\right)^{\left(1-\eta_{k}\right)}$ and $C_{f \ell}^{1}=B \rho_{0} k_{0} \rho_{k+1}^{-1} \times$ $\left(\varepsilon_{k} \rho_{k}^{-1}\right)^{\left(1-\eta_{k}\right)}$, by combining (2.6), (2.7), and a dimensional estimate. Then just as above set $C_{g \ell}=\rho_{0}^{n}\left(\lambda\left(\varepsilon_{0}\right)\right)^{-2} \varepsilon_{k} B^{L_{k}}$ and $C_{g \ell}^{1}=\rho_{0}^{n}\left(\lambda\left(\varepsilon_{0}\right)\right)^{-2} k_{0} \varepsilon_{k} \rho_{k+1}^{-1} B^{L_{k}}$.

Case 2. $\ell \in S^{n}$ for some $n=0, \ldots, k-1$. We must now consider two subcases within this case:

(a) $i \in S^{p}, p=0, \ldots, k$. Set $C_{f \ell}=\rho_{0}\left(\varepsilon_{n} \rho_{n}^{-1}\right)^{\left(1-\eta_{k}\right)}, C_{f \ell}^{1}=B \rho_{0} k_{0} \rho_{k+1}^{-1}\left(\varepsilon_{n} \rho_{n}^{-1}\right)^{\left(1-\eta_{k}\right)}, C_{g \ell}$ $=\rho_{0}^{n}\left(\lambda\left(\varepsilon_{0}\right)\right)^{-2} \varepsilon_{k} B^{L_{k}}$, and $C_{g \ell}^{1}=B \rho_{0}^{n}\left(\lambda\left(\varepsilon_{0}\right)\right)^{-2} k_{0} \rho_{k+1}^{-1} \varepsilon_{k} B^{L_{k}}$.

(b) $i \notin \bigcup_{m=0}^{k} S^{m}$. Set $C_{f \ell}=\rho_{0}\left(\varepsilon_{n} \rho_{n}^{-1}\right)^{\left(1-\eta_{k}\right)}$, and $C_{g \ell}=\rho_{0}^{n}\left(\lambda\left(\varepsilon_{0}\right)\right)^{-2} \varepsilon_{k} B^{L_{k}}$ as before.

Since $\ell \in S^{n}$, and $i \notin \bigcup_{m=0} S^{m}$, we have $|i-\ell|>L_{k}$. Thus by combining (4.15) with

Lemmas 4.1 and 4.2 and our estimates on $\partial g_{\ell} / \partial x_{i}$ above we can pick $C_{f \ell}^{1}=$ $C_{g \ell}^{1}=B \rho_{0}^{n} e^{-m\left(1-\eta_{k}\right)\left(1-6 \beta_{k}\right) L_{k}} \leqq B C_{1} \rho_{0}^{n} \varepsilon_{k+1}$, with $C_{1} \sim \mathcal{O}\left(\left(\varepsilon_{k} \rho_{k}^{-1}\right)^{\alpha}\right)$

With these choices for the constants, the second estimate of (6.3) follows from (5.6) if we set $L=L_{k}$.

Finally we show how (6.8) follows in the case $v=0$. First note that by another application of the fundamental theorem of calculus, one can write

$$
\begin{aligned}
\delta f^{k, \mathrm{r}}\left(I^{\prime}, \phi^{\prime}\right)= & \sum_{\ell=1}^{N} \frac{\partial f^{k, \mathbf{r}}}{\partial I_{\ell}}\left(I^{\prime}, \phi^{\prime}\right) \Xi_{\ell}\left(I^{\prime}, \phi^{\prime}\right)+\sum_{\ell=1}^{N} \frac{\partial f^{k, \mathrm{r}}}{\partial \phi_{\ell}}\left(I^{\prime}, \phi^{\prime}\right) \Delta_{\ell}\left(I^{\prime}, \phi^{\prime}\right) \\
& +\sum_{\ell, p} \int_{0}^{1} d s \int_{0}^{s} d t \frac{\partial^{2} f^{k, \mathrm{r}}}{\partial x_{\ell} \partial x_{p}}\left(x^{\prime}+t g\right) g_{\ell}\left(x^{\prime}\right) g_{p}\left(x^{\prime}\right) .
\end{aligned}
$$

Here, $x$ is as in (1.14), $x^{\prime}$ is as in Lemma 4.2, and $g_{\ell}\left(x^{\prime}\right)$ is either $\Xi_{\ell}\left(x^{\prime}\right)$ or $\Delta_{\ell}\left(x^{\prime}\right)$. We note that the summation over $\ell, p$ here is slightly ambiguous. We emphasize that it should run over both meanings of $x_{\ell}$, and $x_{p}$, i.e. both $I_{\ell}$ and $\phi_{\ell}$, and $I_{p}$ and $\phi_{p}$.

It is straightforward to show that the derivative of the last term with respect to $I_{j}^{\prime}$ is bounded by $\rho_{0}^{n} C_{1} \varepsilon_{k+1}$ on $W\left(r_{k}^{7}, \xi_{k}-\delta-6 ;\left\{I_{k}\right\}\right)$, using Lemma 5.2 and the bounds on $g\left(x^{\prime}\right)$ that we used above. (As before $C_{1} \sim \mathcal{O}\left(\left(\varepsilon_{k} \rho_{k}^{-1}\right)^{\alpha}\right)$.) Since $\Xi_{\ell}\left(I^{\prime}, \phi^{\prime}\right)=$ $\left(\partial S / \partial \phi_{\ell}\right)\left(I^{\prime}, \phi+\Delta\right)$, use the fundamental theorem of calculus to write

$$
\begin{aligned}
& \sum_{\ell=1}^{N} \frac{\partial f^{k, \mathrm{r}}}{\partial I_{\ell}}\left(I^{\prime}, \phi^{\prime}\right) \cdot \Xi_{\ell}\left(I^{\prime}, \phi^{\prime}\right)=\sum_{\ell=1}^{N} \frac{\partial f^{k, \mathrm{r}}}{\partial I_{\ell}}\left(I^{\prime}, \phi^{\prime}\right) \cdot \frac{\partial S}{\partial \phi_{\ell}}\left(I^{\prime}, \phi^{\prime}\right) \\
& \quad+\int_{0}^{1} d s \sum_{\substack{\ell=1 \\
p=1}}^{N} \frac{\partial f^{k, \mathrm{r}}}{\partial I_{\ell}}\left(I^{\prime}, \phi^{\prime}\right) \cdot \frac{\partial^{2} S}{\partial \phi_{p} \partial \phi_{\ell}}\left(I^{\prime}, \phi^{\prime}+s \Delta\right) \cdot \Delta_{p}\left(I^{\prime}, \phi^{\prime}\right) .
\end{aligned}
$$


Because $f^{k, \mathrm{r}}$ and $S$ have no harmonics in common and no contributions from the $v=0$ harmonic, the orthogonality of the trigonometric functions insures that

$$
\int d \phi^{\prime}\left(\sum_{\ell=1}^{N} \frac{\partial f^{k, \mathrm{r}}}{\partial I_{\ell}}\left(I^{\prime}, \phi^{\prime}\right) \cdot \frac{\partial S}{\partial \phi_{\ell}}\left(I^{\prime}, \phi^{\prime}\right)\right)=0 .
$$

Using Lemma 5.2 it is once again easy to show that the derivative of the second term on the right hand side of (6.17), with respect to $I_{j}^{\prime}$ is bounded by $C_{1} \varepsilon_{k+1}$ on $W\left(r_{k}^{7}, \xi_{k}-\delta-6 ;\left\{I_{k}\right\}\right)$. By an exactly analogous procedure we obtain

$$
\left|\sum_{\ell=1}^{N} \int d \phi^{\prime} \frac{\partial}{\partial I_{j}^{\prime}}\left\{\frac{\partial f^{k, \mathrm{r}}}{\partial I_{\ell}}\left(I^{\prime}, \phi^{\prime}\right) \cdot \Delta_{\ell}\left(I^{\prime}, \phi^{\prime}\right)\right\}\right| \leqq C_{1} \varepsilon_{k+1}
$$

on $W\left(r_{k}^{7}, \xi_{k}-\delta-6 ;\left\{I_{k}\right\}\right)$. Putting these observations together we see that

$$
\left|\int d \phi^{\prime} \frac{\partial}{\partial I_{j}^{\prime}}\left\{\delta f^{k, \mathrm{r}}\left(I^{\prime}\right)\right\}\right| \leqq C_{1} \varepsilon_{k+1}
$$

on $W\left(r_{k}^{7}, \xi_{k}-\delta-6 ;\left\{I_{k}\right\}\right)$, and combining this with (6.4), the fact that $0 \notin\left[\bigcup_{m=0}^{k-1}\left(R_{p}^{m} \cup R_{s}^{m}\right)\right] \cup R_{p}^{k}$, and the bounds on $\delta f^{4}, f^{\mathrm{I}}, f^{\mathrm{II}}$, and $f^{\mathrm{III}}$ coming from Proposition 6.1, Proposition 5.1, and Lemmas 5.4 and 5.5 we obtain inequality (6.8) in the case $v=0$.

\section{Some Final Estimates}

We now complete the results that were stated but not proved in Sects. 3 and 4.

We first note that Proposition 3.2 follows from Proposition 4.3. Using (2.5) and (3.2) we readily verify that on $W\left(r_{k}^{1}, \xi_{k}-\delta\right.$; $\left.\left\{I_{k}\right\}\right)$,

$$
\begin{aligned}
& \left|\frac{\partial}{\partial \phi_{j}}\left[\frac{f_{v}^{k}\left(I^{\prime}\right) e^{i v \cdot \phi}}{i\left\langle\omega^{k}\left(I^{\prime}\right), v\right\rangle}\right]\right| \leqq \min \left(B\left(\varepsilon_{k} / \lambda\left(\varepsilon_{0}\right)\right) e^{L_{k}} e^{-(\delta-3 / 2)|v|},\right. \\
& \left.B\left(\rho_{0} / \lambda\left(\varepsilon_{0}\right)\right) e^{L_{k}} e^{-m\left(1-\eta_{k}\right)|j-\ell|} e^{-(\delta-3 / 2)|v|}\right),
\end{aligned}
$$

where $\ell$ is the point in supp $v$ farthest from $j$. This follows by using integration by parts to write the expression for $f_{v}^{k}\left(I^{\prime}\right) v_{j}$ as $-\int d \phi\left(\partial^{2} / \partial \phi_{j} \partial \phi_{\ell}\right)\left\{f^{k}\left(I^{\prime}, \phi\right)\right\} e^{i v \phi} / v_{\ell}$, and the using (2.5) or (2.10), and Cauchy's theorem.

Similarly, if we note that (2.8) and (2.9) imply

$$
\left|i\left\langle\frac{\partial \omega^{k}}{\partial I_{j}^{\prime}}\left(I^{\prime}\right), v\right\rangle\right| \leqq 2|v| e^{-m\left(1-\eta_{k}\right)|j-n|}
$$

where $n$ is the point in supp $v$ closest to $j$, we find

$$
\begin{aligned}
& \left|\frac{\partial}{\partial I_{j}^{\prime}}\left[\frac{f_{v}\left(I^{\prime}\right) e^{i v \cdot \phi}}{i\left\langle\omega^{k}\left(I^{\prime}\right), v\right\rangle}\right]\right| \leqq \min \left(B\left(\varepsilon_{k} / \lambda^{2}\left(\varepsilon_{0}\right)\right) \rho_{0}^{-1} e^{2 L_{k}}|v| e^{(3-\delta)|v|},\right. \\
& \left.\quad\left(B / \lambda^{2}\left(\varepsilon_{0}\right)\right) e^{-m\left(1-\eta_{k}\right)|j-\ell|} e^{2 L_{k}}|v| e^{(3-\delta)|v|}\right),
\end{aligned}
$$

again on $W\left(r_{k}^{1}, \xi_{k}-\delta ;\left\{I_{k}\right\}\right)$. If we now apply Proposition 4.3 with $K_{1}=$ $\rho_{0}^{n}\left(B / \lambda^{2}\left(\varepsilon_{0}\right)\right) e^{2 L_{k}}, e(i)=\varepsilon_{k} \rho_{0}^{-1}, \kappa_{1}=m\left(1-\eta_{k}\right)$, and $\delta^{\prime}=\delta / 2,($ which implies $\delta \geqq 12)$ 
we have

$$
\sup \left|\frac{\partial S}{\partial y_{j}}\right| \leqq \rho_{0}^{n}\left(B / \lambda^{2}\left(\varepsilon_{0}\right)\right) e^{2 L_{k}} \varepsilon_{k} \rho_{0}^{-1} B^{\prime 2 L_{k}} \leqq\left(\rho_{0}^{n} / \lambda^{2}\left(\varepsilon_{0}\right)\right) \varepsilon_{k} B^{L_{k}},
$$

on $W\left(r_{k}^{1}, \xi_{k}-\delta\right.$; $\left.\left\{I_{k}\right\}\right)$, by (4.7), where $y \in \mathbb{C}^{2 N}$ is defined in Proposition 3.2. This verifies the first inequality in (3.7).

Next note that using (2.10), Cauchy's theorem, and a dimensional estimate we have

$$
\begin{aligned}
& \left|\frac{\partial^{2}}{\partial \phi_{i} \partial \phi_{j}}\left[\frac{f_{v}^{k}\left(I^{\prime}\right) e^{i v \cdot \phi}}{i\left\langle\omega^{k}\left(I^{\prime}\right), v\right\rangle}\right]\right| \leqq \min \left(\left(B \rho_{0} / \lambda\left(\varepsilon_{0}\right)\right) e^{-m\left(1-\eta_{k}\right)|i-j|} e^{L_{k} \cdot|v|^{2} e^{(3 / 2-\delta)|v|}}\right. \\
& \left.\quad\left(B \rho_{0} / \lambda\left(\varepsilon_{0}\right)\right) e^{-m\left(1-\eta_{k}\right)|i-l|} e^{L_{k}}|v|^{2} e^{(3 / 2-\delta)|v|}\right)
\end{aligned}
$$

on $W\left(r_{k}^{1}, \xi_{k}-\delta-1 ;\left\{I_{k}\right\}\right)$, where as usual, $\ell$ is the site in supp $v$ most distant from $i$. Similarly

$$
\begin{aligned}
& \left|\frac{\partial^{2}}{\partial \phi_{i} \partial I_{j}^{\prime}}\left(\frac{f_{v}\left(I^{\prime}\right) e^{l v \cdot \phi}}{i\left\langle\omega^{k}\left(I^{\prime}\right), v\right\rangle}\right)\right| \leqq \min \left(\left(B / \lambda^{2}\left(\varepsilon_{0}\right)\right) e^{-m\left(1-\eta_{k}\right)|i-j|}|v|^{2} e^{2 L_{k}} e^{(3-\delta)|v|},\right. \\
& \left.\quad B\left[\left(\rho^{k}\right)_{j}-\left(i_{k}^{*}\right)_{j}\right]^{-1}\left(\rho_{0} / \lambda^{2}\left(\varepsilon_{0}\right)\right) e^{-m\left(1-\eta_{k}\right)|i-l|}|v|^{2} e^{2 L_{k}} e^{(3-\delta)|v|}\right)
\end{aligned}
$$

and

$$
\begin{aligned}
& \left|\frac{\partial^{2}}{\partial I_{i}^{\prime} \partial I_{j}^{\prime}}\left(\frac{f_{v}\left(I^{\prime}\right) e^{i v \cdot \phi}}{i\left\langle\omega^{k}\left(I^{\prime}\right), v\right\rangle}\right)\right| \leqq \min \left(\left(B / \rho_{0} \lambda^{3}\left(\varepsilon_{0}\right)\right) e^{-m\left(1-\eta_{k}\right)|1-j|}|v|^{2} e^{3 L_{k}} e^{(5-\delta)|v|},\right. \\
& \left.\cdot\left(B / \lambda^{3}\left(\varepsilon_{0}\right)\right)\left[\left(\rho^{k}\right)_{j}-\left(r_{k}^{1}\right)_{j}\right]^{-1} \rho_{0} e^{-m\left(1-\eta_{k}\right)|t-\ell|}|v|^{2} e^{3 L_{k}} e^{(5-\delta)|v|}\right),
\end{aligned}
$$

on $W\left(r_{k}^{1}, \xi_{k}-\delta ;\left\{I_{k}\right\}\right)$. If we combine (7.5)-(7.7) with the observation that if $\chi<$ $\min \left(C_{1}, C_{2}\right)$, then $\chi<C_{1}^{\beta} C_{2}^{(1-\beta)}$ for $\beta \in[0,1]$, we see that we can apply the second half of Proposition 4.5, choosing

$$
\begin{aligned}
& K_{2}=K_{3}=B\left\{\rho_{0}^{-1}\left[\left(\rho^{k}\right)_{j}-\left(r_{k}^{1}\right)_{j}\right]\right\}^{-\beta_{k}} \cdot\left(\lambda\left(\varepsilon_{0}\right)\right)^{-3} e^{-m\left(1-\eta_{k}\right)\left(1-\beta_{k}\right)|t-\jmath|} e^{3 L_{k}}, \\
& \kappa_{2}=m\left(1-\eta_{k}\right) \beta_{k}, \text { and } \delta^{\prime}=\delta / 2 .
\end{aligned}
$$

Then (4.9) implies that

$$
\sup \left|\frac{\partial^{2} S}{\partial y_{i} \partial y_{j}}(y)\right| \leqq B \rho_{0}^{n}\left\{\rho_{0}^{-1}\left[\left(\rho^{k}\right)_{j}-\left(r_{k}^{1}\right)_{j}\right]\right\}^{-\beta_{k}}\left(\lambda\left(\varepsilon_{0}\right)\right)^{-3} e^{3 L_{k}} B^{\prime \prime|l-j|} e^{-m\left(1-\eta_{k}\right)|i-j|},
$$

with the supremum running over $W\left(r_{k}^{1}, \xi_{k}-\delta ;\left\{I_{k}\right\}\right)$. Note that we can assume $|i-j|>\left(L_{k} / 4\right)$, since otherwise the second estimate in (3.7) follows from the first by a dimensional estimate. But then, using the fact that

$$
B\left\{\rho_{0}^{-1}\left[\left(\rho^{k}\right)_{j}-\left(r_{k}^{1}\right)_{j}\right]\right\}^{-\beta_{k}}\left(\lambda\left(\varepsilon_{0}\right)\right)^{-3} e^{3 L_{k}} B^{\prime \prime|l-J|} e^{-m\left(1-\eta_{k}\right) \beta_{k}|i-j|} \leqq 1,
$$

when $|i-j|>\left(L_{k} / 4\right)$, we obtain the second estimate of (3.7).

We next prove Proposition 4.3. To prove (4.7) note that

$$
\left|\frac{\partial g}{\partial x_{1}}(x)\right| \leqq \sum_{\substack{v_{i} \\|1-\ell| \leqq L}}\left|\frac{\partial g_{v}}{\partial x_{i}}\left(x^{\prime}\right)\right|+\sum_{\substack{v: \\|i-\ell|>L}}\left|\frac{\partial g_{v}}{\partial x_{i}}(x)\right|
$$


where $L$ is any positive integer and $\ell$ is the point in supp $v$ such that $|i-\ell|$ is maximized. Bound the summand in the first term on the right hand side of (7.10) by $K_{1} e(i) e^{-\delta^{\prime}|\nu|}$, and in the second term bound it by $K_{1} e^{-\kappa_{1}|t-\ell|} e^{-\delta^{\prime}|v|}$. Next note that the number of vectors with $|v|=M$ and $|i-\ell|=\widetilde{L}$ is bounded by $2(2 \widetilde{L}+1) 2^{\tilde{L}} 2^{2 M}$. This estimate follows from the observation that the number of vectors with $|v|=M$, $d(\operatorname{supp} v)=L^{\prime}$, and the rightmost point in $\operatorname{supp} v$ fixed is bounded by $2^{L^{\prime}} 2^{2 M}$ (a discussion of this bound is contained in [6]) and then noting that if $|i-\ell|=\tilde{L}$ one must have $0 \leqq L^{\prime} \leqq 2 \tilde{L}$, and that there are at most $2 \tilde{L}+1$ choices for the rightmost point in $\operatorname{supp} v$. Thus (7.9) is bounded by

$$
\begin{aligned}
& \sum_{M=0}^{\infty} \sum_{\tilde{L}=0}^{L} 2(2 \tilde{L}+1) 2^{L} 2^{2 M} K_{1} e(i) e^{-\delta^{\prime} M}+\sum_{M=0}^{\infty} \sum_{\tilde{L}=L+1}^{\infty} 2(2 \tilde{L}+1) 2^{L} 2^{2 M} K_{1} e^{-\kappa_{1}} \tilde{L}^{-\delta^{\prime} M} \\
& \quad \leqq 2^{2}(2 L+1) 2^{L} K_{1} e(i)+2^{4} K_{1} e^{-\left(\kappa_{1}-2 \ln 2\right) L}
\end{aligned}
$$

from which (4.7) follows immediately if $L>\left(2 / \kappa_{1}\right)|\ln e(i)|$.

Inequality (4.9) is proved in much the same fashion. Note that

$$
\left|\frac{\partial^{2} g}{\partial x_{i} \partial x_{j}}(x)\right| \leqq \sum_{\substack{v: \\|\imath-\ell| \leqq|t-j|}}\left|\frac{\partial^{2} g_{v}}{\partial x_{i} \partial x_{j}}\right|+\sum_{\substack{v: \\|i-\ell|>|i-j|}}\left|\frac{\partial^{2} g_{v}}{\partial x_{i} \partial x_{j}}\right|
$$

Bound the first sum by

$$
\sum_{M=0}^{\infty} \sum_{\mathcal{L}=0}^{|i-j|} 2(2 \tilde{L}+1) 2^{\tilde{L}} 2^{2 M} K_{2} e^{-\kappa_{2}|i-j|} e^{-\delta^{\prime} M} \leqq K_{2} B^{(|t-j|+1)} e^{-\kappa_{2}|t-j|} .
$$

Bound by the second term by

$$
\sum_{M=0}^{\infty} \sum_{\mathcal{L}=|\imath-\jmath|+1}^{\infty} 2(2 \widetilde{L}+1) 2^{\mathcal{L}} 2^{2 M} K_{3} e^{-\kappa_{2} \mathcal{L}} e^{-\delta^{\prime} M} \leqq K_{3} B^{(|\imath-\jmath|+1)} e^{-\kappa_{2}|i-j|},
$$

and (4.9) follows immediately by combining (7.12) and (7.13). lemma:

We finish up by proving Lemma 4.2. As in [6] the proof turns on the following

Lemma 7.1. Let $M$ be an $n \times n$ matrix whose elements satisfy $\left|M_{i j}\right| \leqq \min (c$, $\left.c_{1} e^{-\kappa|t-j|}\right)$. If $c$ and $c_{1}$ are less than (1/4), and $\kappa \geqq 2 \ln 2$ then

$$
\left|(0-M)_{i j}^{-1}-\delta_{i j}\right| \leqq \min \left(c B^{L}, 2^{4} 2^{|i-j|} e^{-\kappa|t-j|}\right),
$$

where $B$ is some universal constant, and $\bar{L}$ is any number such that $\bar{L}>(2 / \kappa)|\ln c|$.

The proof of this lemma is in Appendix B.

Let $\tilde{y}$ be the holomorphic map taking $W\left(r_{k}^{4}, \xi_{k}-\delta-3 ;\left\{I_{k}\right\}\right)$ into $W\left(r_{k}^{3}, \xi_{k}-\delta\right.$ -2 ; $\left.\left\{I_{k}\right\}\right)$ defined by $\tilde{y}_{i}\left(x^{\prime}\right)=x_{i}^{\prime}$ if $1 \leqq i \leqq N$, and $y_{i}\left(x^{\prime}\right)=\tilde{x}_{i}\left(x^{\prime}\right)$ if $N<i \leqq 2 N$, where $\tilde{x}_{i}\left(x^{\prime}\right)=\left\{I_{i}\left(I^{\prime}, \phi^{\prime}\right)\right.$ if $1 \leqq i \leqq N$ and $\phi_{i-N}\left(I^{\prime}, \phi^{\prime}\right)$ if $\left.N<i \leqq 2 N\right\}$ and $x_{i}^{\prime}=\left\{I_{i}^{\prime}\right.$ if $1 \leqq i \leqq N$ and $\phi_{i-N}^{\prime}$ if $\left.N<i \leqq 2 N\right\}$. Finally let $y_{i}=\left\{I_{i}^{\prime}\right.$ if $1 \leqq i \leqq N, \phi_{i-N}$ if $\left.N<I \leqq 2 N\right\}$. This notation saves writing out many special cases, but it has the following disadvantage. If we consider $\partial^{2} S / \partial I_{j}^{\prime} \partial \phi_{k}\left(I^{\prime}, \phi^{\prime}\right)=\partial^{2} S / \partial y_{j} \partial y_{\ell}(y)$, where $\ell=k+N$, we expect it to decay only as $\exp \left[-m\left(1-\eta_{k}\right)|j-k|\right]$, not as $\exp \left[-m\left(1-\eta_{k}\right)|j-\ell|\right]$. Thus we define $\delta(j, \ell)=|(j-\ell)(\bmod N)|$, and then we see that the derivatives above decay as $\exp \left[-m\left(1-\eta_{k}\right) \delta(j, \ell)\right]$.

If $N<i \leqq 2 N, \tilde{x}_{i}\left(x^{\prime}\right)=x_{i}^{\prime}-\partial S / \partial y_{i-N} \circ \tilde{y}\left(x^{\prime}\right)$, by (3.14), and (3.11), where $y$ is as in 
Proposition 3.2. Thus,

$$
\begin{aligned}
& \frac{\partial \tilde{x}_{i}}{\partial x_{j}^{\prime}}\left(x^{\prime}\right)=\delta_{i j}-\sum_{\ell=1}^{2 N} \frac{\partial^{2} S}{\partial y_{i-N} \partial y_{\ell}} \circ \tilde{y}\left(x^{\prime}\right) \cdot \frac{\partial \tilde{y}_{\ell}}{\partial x_{j}^{\prime}}\left(x^{\prime}\right) \\
& =\delta_{i j}-\sum_{\ell=1}^{N}\left(\frac{\partial^{2} S}{\partial y_{i-N} \partial y_{\ell}} \circ \tilde{y}\left(x^{\prime}\right)\right) \cdot \delta_{\ell j}
\end{aligned}
$$

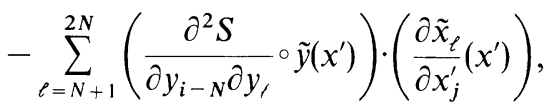

where the first of these steps used the chain rule, and the second the definition of $\tilde{y}$. Let $M$ be the $N \times N$ matrix with elements $M_{i j}=\left(-\partial^{2} S / \partial y_{i} \partial y_{j+N}\right) \circ \tilde{y}\left(x^{\prime}\right)$. We now consider two cases. If the $j$ in (7.15) is less than or equal to $N$, define $D$ to be the $N \times N$ matrix with elements $D_{i j}=\left(\partial \tilde{x}_{i+N} / \partial x_{j}^{\prime}\right)\left(x^{\prime}\right)$, and $\Lambda$ the $N \times N$ matrix with elements $\Lambda_{i j}=-\left(\partial^{2} S / \partial y_{i} \partial y_{j}\right) \circ \tilde{y}\left(x^{\prime}\right)$. If the $j$ in (7.15) is greater than $N$, let $D^{\prime}$ be the matrix with elements $D_{i j}^{\prime}=\left(\partial \tilde{x}_{i+N} / \partial x_{j+N}^{\prime}\right)\left(x^{\prime}\right)$, and $\Lambda^{\prime}=N \times N$ identity matrix. Then, (7.15) becomes the pair of matrix equations

$$
D=\Lambda+M D \quad \text { and } \quad D^{\prime}=\Lambda^{\prime}+M D^{\prime}
$$

or

$$
D=(\mathbb{\square}-M)^{-1} \Lambda \quad \text { and } \quad D^{\prime}=(\mathbb{\square}-M)^{-1} \Lambda^{\prime} .
$$

By Proposition 3.2 and a dimensional estimate,

$$
\left|M_{i j}\right| \leqq \min \left(\rho_{0}^{n}\left(\lambda\left(\varepsilon_{0}\right)\right)^{-2} k_{0} \varepsilon_{k} \rho_{k+1}^{-1} B^{L_{k}}, \rho_{0}^{n} e^{-m\left(1-\eta_{k}\right)\left(1-\beta_{k}\right)|t-\jmath|}\right)
$$

for all $x^{\prime}$ in $W\left(r_{k}^{4}, \xi_{k}-\delta-3 ;\left\{I_{k}\right\}\right)$. Note that if $i$ and $j$ are both greater than $N$, the second equation in (7.17) implies

$$
\frac{\partial \tilde{x}_{i}}{\partial x_{j}^{\prime}}\left(x^{\prime}\right)=\left[(\mathbb{\square}-M)^{-1}\right]_{i-N, j-N},
$$

and (4.4) follows (in the case $i, j>N$ ) from Lemma 7.1. If $i$ is greater than $N$ and $j$ is less than or equal to $N,(7.17)$ implies

$$
\frac{\partial \tilde{x}_{i}}{\partial x_{j}^{\prime}}\left(x^{\prime}\right)=\sum_{l=1}^{N}(\square-M)_{i-N, \ell}^{-1} \Lambda_{\ell j} .
$$

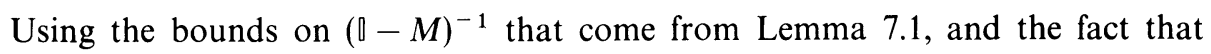
Proposition 3.2 implies

$$
\left|\Lambda_{\ell j}\right| \leqq \min \left(\rho_{0}^{n}\left(\lambda\left(\varepsilon_{0}\right)\right)^{-2} k_{0} \varepsilon_{k} \rho_{k+1}^{-1} B^{L_{k}}, \rho_{0}^{n} e^{\left.-m\left(1-\eta_{k}\right)\left(1-\beta_{k}\right) \delta(\ell, j)\right)}\right)
$$

for all $x^{\prime}$ in $W\left(r_{k}^{4}, \xi_{k}-\delta-3 ;\left\{I_{k}\right\}\right)$, it is easy to bound (7.19) by

$$
\begin{aligned}
\left|\frac{\partial \tilde{x}_{i}}{\partial x_{j}^{\prime}}\left(x^{\prime}\right)\right| \leqq & \min \left(B^{\prime} \rho_{0}^{n}\left(\lambda\left(\varepsilon_{0}\right)\right)^{-2} k_{0} \varepsilon_{k} \rho_{k+1}^{-1} B^{L_{k}}, B \rho_{0}^{n}(\delta(i, j)+1) 2^{\delta(i, j)}\right. \\
& \left.\cdot e^{-m\left(1-\eta_{k}\right)\left(1-\beta_{k}\right) \delta(i, j)}\right) .
\end{aligned}
$$

from which (4.4) (in the case $j \leqq N, i>N$ ) follows. 
If $1 \leqq i \leqq N, \tilde{x}_{i}\left(x^{\prime}\right)=x_{i}+\left(\partial S / \partial y_{i+N}\right) \circ \tilde{y}\left(x^{\prime}\right)$, so applying the chain rule we obtain

$$
\begin{aligned}
\frac{\partial \tilde{x}_{i}}{\partial x_{j}^{\prime}}\left(x^{\prime}\right)-\delta_{i j}= & \sum_{\ell=1}^{N}\left[\frac{\partial^{2} S}{\partial y_{i+N} \partial y_{\ell}} \circ \tilde{y}\left(x^{\prime}\right)\right] \cdot \delta_{\ell j} \\
& +\sum_{\ell=N+1}^{2 N}\left[\frac{\partial^{2} S}{\partial y_{i+N} \partial y_{\ell}} \circ \tilde{y}\left(x^{\prime}\right)\right] \cdot \frac{\partial \tilde{x}_{\ell}}{\partial x_{j}^{\prime}}\left(x^{\prime}\right) .
\end{aligned}
$$

Use Proposition 3.2 to bound derivatives of $S$, and use (7.18) and (7.20) to bound $\partial \tilde{x}_{\ell} / \partial x_{j}^{\prime}$, for $N<\ell \leqq 2 N$. If we do so we find (on $W\left(r_{k}^{4}, \xi_{k}-\delta-3 ;\left\{I_{k}\right\}\right)$ ).

$$
\begin{aligned}
\left|\frac{\partial \tilde{x}_{i}}{\partial x_{j}^{\prime}}\left(x^{\prime}\right)-\delta_{i j}\right| \leqq & \min \left(\rho_{0}^{n} k_{0} \varepsilon_{k} \rho_{k+1}^{-1} B^{L_{k}} /\left(\lambda\left(\varepsilon_{0}\right)\right)^{2}, B \rho_{0}^{n}(\delta(i, j)+1)\right. \\
& \left.\cdot 2^{|\imath-\jmath|} e^{-m\left(1-\eta_{k}\right)\left(1-\beta_{k}\right) \delta(i, j)}\right) .
\end{aligned}
$$

The remaining cases of (4.4) follow from (7.22).

Finally, we turn to the proof of (4.5). If $N<\ell \leqq 2 N, \quad \tilde{x}_{\ell}\left(x^{\prime}\right)=x_{\ell}^{\prime}-$ $\left(\partial S / \partial y_{\ell-N}\right) \circ \tilde{y}\left(x^{\prime}\right)$, and the chain rule and the definition of $\tilde{y}$ imply

$$
\begin{aligned}
\frac{\partial^{2} \tilde{x}_{\ell}}{\partial x_{i}^{\prime} \partial x_{j}^{\prime}}\left(x^{\prime}\right)= & -\sum_{p, n=1}^{2 N}\left[\frac{\partial^{3} S}{\partial y_{\ell-N} \partial y_{p} \partial y_{n}} \circ \tilde{y}\left(x^{\prime}\right)\right]\left[\frac{\partial \tilde{y}_{p}}{\partial x_{j}^{\prime}}\left(x^{\prime}\right)\right]\left[\frac{\partial \tilde{y}_{n}}{\partial x_{i}^{\prime}}\left(x^{\prime}\right)\right] \\
& -\sum_{n=N+1}^{2 N}\left[\frac{\partial^{2} S}{\partial y_{\ell-N} \partial y_{n}} \circ \tilde{y}\left(x^{\prime}\right)\right]\left[\frac{\partial^{2} \tilde{x}_{n}}{\partial x_{i}^{\prime} \partial x_{j}^{\prime}}\left(x^{\prime}\right)\right] .
\end{aligned}
$$

Assume for the moment that $j$ is less than or equal to $N$. Define $D^{2}$ to be the $N \times N$ matrix with elements $D_{\ell j}^{2}=\left(\partial^{2} \tilde{x}_{\ell+N} / \partial x_{i}^{\prime} \partial x_{j}^{\prime}\right)\left(x^{\prime}\right)$, and $\Lambda^{2}$ the $N \times N$ matrix with elements

$$
\Lambda_{p j}^{2}=\sum_{q, n=1}^{2 N}\left[\frac{\partial^{3} S}{\partial y_{p} \partial y_{q} \partial y_{n}} \circ \tilde{y}\left(x^{\prime}\right)\right]\left[\frac{\partial \tilde{y}_{q}}{\partial x_{j}^{\prime}}\left(x^{\prime}\right)\right]\left[\frac{\partial \tilde{y}_{n}}{\partial x_{i}^{\prime}}\left(x^{\prime}\right)\right] .
$$

If $M$ is the same matrix as in (7.16), then (7.23) may be rewritten as

$$
D^{2}=\Lambda^{2}+M D^{2} \text { or } D^{2}=(\mathbb{\square}-M)^{-1} \Lambda^{2} \text {. }
$$

Using Proposition 3.2, a dimensional estimate, and (4.4) it is easy to show that for $x^{\prime}$ in $W\left(r_{k}^{4}, \xi_{k}-\delta-3 ;\left\{I_{k}\right\}\right)$,

$$
\left|\Lambda_{p j}^{2}\right| \leqq B \rho_{0}^{n}\left[\left(r_{k}^{2}\right)_{p}-\left(r_{k}^{3}\right)_{p}\right]^{-1} e^{-m\left(1-\eta_{k}\right)\left(1-4 \beta_{k}\right) \delta(i, j)} .
$$

Using this bound, and the bound on $(\square-M)^{-1}$ that comes from Lemma 7.1 (and which we used previously in (7.18)), we find

$$
\begin{aligned}
\left|\frac{\partial^{2} \tilde{x}_{\ell}}{\partial x_{i}^{\prime} \partial x_{j}^{\prime}}\left(x^{\prime}\right)\right| & \leqq \sum_{n}\left|(\mathbb{0}-M)_{\hat{\ell} n}^{-1}\right| \Lambda_{n j}^{2} \mid \\
& \leqq B \rho_{0}^{n}\left[\left(r_{k}^{2}\right)_{\hat{\ell}}-\left(r_{k}^{3}\right)_{\hat{\ell}}\right]^{-1} e^{-m\left(1-\eta_{k}\right)\left(1-4 \rho_{k}\right) \delta(i, j)}
\end{aligned}
$$

where $\hat{\ell}=\ell(\bmod N)$. The bound in the case $j>N$ follows in analogous fashion, and we don't write out the details. This completes the proof of (4.5) for $\ell>N$. 
If $1 \leqq \ell \leqq N$, then the definition of $\tilde{x}_{\ell}\left(x^{\prime}\right)$, and the chain rule imply

$$
\begin{aligned}
\frac{\partial^{2} \tilde{x}_{\ell}}{\partial x_{i}^{\prime} \partial x_{j}^{\prime}}\left(x^{\prime}\right)= & \sum_{p, q=1}^{2 N}\left[\frac{\partial^{2} S}{\partial y_{\ell+N} \partial y_{p} \partial y_{q}} \circ \tilde{y}\left(x^{\prime}\right)\right]\left[\frac{\partial \tilde{y}_{p}}{\partial x_{j}^{\prime}}\left(x^{\prime}\right)\right]\left[\frac{\partial \tilde{y}_{q}}{\partial x_{i}^{\prime}}\left(x^{\prime}\right)\right] \\
& +\sum_{p=N+1}^{2 N}\left[\frac{\partial^{2} S}{\partial y_{\ell+N} \partial y_{p}} \circ \tilde{y}\left(x^{\prime}\right)\right]\left[\frac{\partial^{2} \tilde{x}_{p}}{\partial x_{i}^{\prime} \partial x_{j}^{\prime}}\left(x^{\prime}\right)\right] .
\end{aligned}
$$

Bound derivatives of $S$ by Proposition 3.2, bound $\partial^{2} \tilde{x}_{p} / \partial x_{i}^{\prime} \partial x_{j}^{\prime}$ for $p>N$ by (7.25), and bound derivatives of $\tilde{y}$ by noting that $\partial \tilde{y}_{p} / \partial x_{j}^{\prime}=\delta_{p j}$ if $p \leqq N$ and $\partial \tilde{y}_{p} / \partial x_{j}^{\prime}=$ $\partial \tilde{x}_{p} / \partial x_{j}^{\prime}$ if $p>N$, and then apply (4.4). Combining these observations yields the remaining cases of (4.5).

\section{Appendix A. The Inverse Function Theorem}

We wish to invert the equations

$$
\begin{aligned}
I & =I^{\prime}+\frac{\partial S}{\partial \phi}\left(I^{\prime}, \phi\right), \\
\phi^{\prime} & =\phi+\frac{\partial S}{\partial I^{\prime}}\left(I^{\prime}, \phi\right) .
\end{aligned}
$$

Standard analytic inverse function theorems guarantee that these maps can be inverted if they are $1-1$. Assume there exists $I^{1}$ and $I^{2}$ in $W\left(r_{k}^{2}, \xi_{k}-\delta-1 ;\left\{I_{k}\right\}\right)$, with

$$
I^{1}+\frac{\partial S}{\partial \phi}\left(I^{1}, \phi\right)=I^{2}+\frac{\partial S}{\partial \phi}\left(I^{2}, \phi\right) .
$$

There is a path $\gamma$, contained in $W\left(r_{k}^{2}, \xi_{k}-\delta-1 ;\left\{I_{k}\right\}\right)$, joining $I^{1}$ to $I^{2}$ consisting of $N$ pieces, $\gamma_{j}$, along which only the $j^{\text {th }}$ coordinate of $I$ varies, and the length of $\gamma_{j}$ is $\left|I_{j}^{1}-I_{j}^{2}\right|$. Then the fundamental theorem of calculus implies

$$
\sum_{i=1}^{N}\left|I_{i}^{1}-I_{i}^{2}\right| \leqq \sum_{i, j}\left|\int_{\gamma_{j}} d I_{j}^{\prime \prime} \frac{\partial^{2} S}{\partial \phi_{i} \partial I_{j}^{\prime}}\left(I^{\prime \prime}, \phi\right)\right| .
$$

But if $\sup \sum_{i}\left|\left(\partial^{2} S / \partial \phi_{i} \partial I_{j}^{\prime}\right)\right| \leqq(1 / 2)$ on $W\left(r_{k}^{2}, \xi_{k}-\delta-1 ;\left\{I_{k}\right\}\right)$, for all $j$, this last sum is bounded by $(1 / 2) \sum_{j}\left|I_{j}^{1}-I_{j}^{2}\right|$, implying $I^{1}=I^{2}$. Thus, the first equation in (A.1) is invertible as $I^{\prime}=I+\Xi(I, \phi)$ on the image of $W\left(r_{k}^{2}, \xi_{k}-\delta-1 ;\left\{I_{k}\right\}\right)$. If $\sup \left|\left(\partial S / \partial \phi_{i}\right)\left(I^{\prime}, \phi\right)\right|<c_{1} \rho_{k+1} / k_{0}$ for all $i$, the range of the map must contain $W\left(r_{k}^{3}, \xi_{k}-\delta-1 ;\left\{I_{k}\right\}\right)$, and hence $\Xi^{\prime}(I, \phi)$ is analytic on this domain.

To invert the second equation in (A.1) assume there are $\phi^{1}$ and $\phi^{2}$ in $W\left(r_{k}^{2}, \xi_{k}-\right.$ $\left.\delta-1 ;\left\{I_{k}\right\}\right)$ such that

$$
\phi^{1}+\frac{\partial S}{\partial I^{\prime}}\left(I^{\prime}, \phi^{1}\right)=\phi^{2}+\frac{\partial S}{\partial I^{\prime}}\left(I^{\prime}, \phi^{2}\right)
$$

Pick, $\gamma$, a path contained in $W\left(r_{k}^{2}, \xi_{k}-\delta-1 ;\left\{I_{k}\right\}\right)$, joining $\phi^{1}$ to $\phi^{2}$, and consisting of $N$ pieces, $\gamma_{j}$, along which only one component of $\phi$ varies. As before $\gamma_{j}$ may be picked 
so that the length of $\gamma_{j}$ is $\left|\phi_{j}^{1}-\phi_{j}^{2}\right|$, and applying the fundamental theorem of calculus just as above we find that the map is $1-1$ if $\sup \sum_{i}\left|\left(\partial^{2} S / \partial I_{i}^{\prime} \partial \phi_{j}\right)\right| \leqq(1 / 2)$ for all $j$ on $W\left(r_{k}^{2}, \xi_{k}-\delta-1 ;\left\{I_{k}\right\}\right)$. Thus, the second equation in (A.1) can be inverted as

$$
\phi=\phi^{\prime}+\Delta\left(I^{\prime}, \phi^{\prime}\right)
$$

with $\Delta\left(I^{\prime}, \phi^{\prime}\right)$ analytic on the range of the original map. If $\sup \mid\left(\partial S / \partial I_{j}^{\prime} \mid<(1 / 2)\right.$ for all $j$ (with the supremum running over $\left.W\left(r_{k}^{2}, \xi_{k}-\delta-1 ;\left\{I_{k}\right\}\right)\right), \Delta\left(I^{\prime}, \phi^{\prime}\right)$ must be analytic at least on $W\left(r_{k}^{2}, \xi_{k}-\delta-2 ;\left\{I_{k}\right\}\right)$.

\section{Appendix B. The Proof of Lemma 7.1}

Using the random walk expansion of [2] we write

$$
(\square-M)_{i j}^{-1} \mid=\sum_{\Omega: i \rightarrow j} \Lambda_{j j}^{-1}\left(\prod_{\ell \in L} \Lambda_{\ell \ell}^{-n(\ell, \Omega)}\right)\left(\prod_{s \in \Omega} \tilde{M}_{s}\right) .
$$

On the right-hand side of (A.1), $\Lambda_{j j}=1-M_{j j}$ and $\Omega$ is a random walk on the lattice $L=\{1, \ldots, N\}$, i.e. a set of pairs $\left\{\left(i_{1}, i_{2}\right), \ldots,\left(i_{k}, i_{k+1}\right)\right\}, i_{j} \in\{1, \ldots, N\}$. Each of the pairs is referred to as a step, $s$, with $|\Omega|$ the number of steps in the walk, and $\Omega$ : $i \rightarrow j$ means $i_{1}=i, i_{k+1}=j$. Finally $\tilde{M}_{s}=\tilde{M}_{i_{j}, i_{j+1}}=\left(1-\delta_{i_{j} l_{j+1}}\right) M_{i_{j} i_{j+1}},|s|=\left|i_{j+1}-i_{j}\right|$, and $n(j, \Omega)$ is the number of times $j$ appears as the first element in some step in $\Omega$.

The second bound on the right hand side of (7.14) follows by estimating the sum on the right hand side of (B.1) exactly as was done in proving Lemma 2.6 in [7], so we don't repeat that here.

The other bound in (7.14) follows by noting that if $N=|\Omega|$, and $L=\sum_{s \in \Omega}|s|$, there is exactly one walk with $M=0$, and its contribution to the sum is $\Lambda_{j j} \delta_{i j}$. Every walk which contributes to (B.1) must have $L \geqq|i-j|$. Furthermore, since any walk with a step of zero length gives no contribution to the $\operatorname{sum}\left(\tilde{M}_{j j}=0\right)$, there are at most $2^{L} 2^{N}$ walks with fixed $L$. We bound factors of $\left|\Lambda_{j j}\right|^{-1}$ by $(1-c)^{-1}$, and factors of $\prod_{s \in \Omega}\left|M_{s}\right|$ by $c^{N}$ if $L(\Omega) \leqq \tilde{L}$, or by $c_{1}^{N} e^{-\kappa L(\Omega)}$ if $L(\Omega)>\bar{L}$, where $\bar{L}$ is defined in (7.14). Thus,

$$
\begin{aligned}
\left|(\mathbb{Q}-M)^{-1}{ }_{i j}-\delta_{i j}\right| \leqq & \delta_{i j}\left|\left(1-\Lambda_{j j}\right)\right|+\sum_{M=1}^{\infty} \sum_{L=|i-j|}^{L}(1-c)^{-(N+1)} c^{N} 2^{L} 2^{N} \\
& +\sum_{N=1}^{\infty} \sum_{L=\tilde{L}+1}^{\infty}(1-c)^{-(N+1)} c_{1}^{N} e^{-\kappa L} 2^{L} 2^{N},
\end{aligned}
$$

and the first bound on the right hand side of (7.14) follows by summing the geometric series.

Acknowledgements. I wish to thank G. Gallavotti, R. de la Llave, and L. Thomas for helpful conversations and L. Galgani for communicating his results to me before publication. I wish also to thank the Department of Mathematics, University of Virginia for its hospitality while much of this work was completed. 


\section{References}

1. Benettin, G., Galgani, L., Giorgilli, A.: Classical perturbation theory for systems of weakly coupled rotators. Preprint

2. Brydges, D., Fröhlich, J., Spencer, T.: The random walk representation of classical spin systems and correlation inequalities. Commun. Math. Phys. 83, 123 (1982)

3. Chierchia, L., Gallavotti, G.: Smooth prime integrals for quasi-Hamiltonian systems. Il Nuovo Cimento 67B, 277 (1982)

4. Gallavotti, G.: Perturbation theory for classical Hamiltonian systems. In: Scaling and self-similarity in physics. Fröhlich J. (ed.) Boston, MA: Birkhäuser Boston 1983

5. Nekhoroshev, N.: An exponential estimate of the time of stability of nearly-integrable Hamiltonian systems. Russ. Math. Surv. 32, 1 (1977)

6. Wayne, C. E.: The KAM theory of systems with short range interactions, I. Commun. Math. Phys. 96, $311(1984)$

7. Wayne, C. E.: The KAM theory of systems with short range interactions, II. Commun. Math. Phys. 96, $331(1984)$

8. Wayne, C. E.: Bounds on the trajectories of a system of weakly coupled rotators. Commun. Math. Phys. (in press)

Communicated by J. L. Lebowitz

September 14, 1984; in revised form April 1, 1985 